\title{
Modeling And Simulation of The Advanced Structured Surfaces Machined By Specially Patterned Grinding Wheels Via The Structuring Grinding Process
}

\author{
Amr Monier \\ Harbin Institute of Technology \\ Bing Guo ( $\sim$ guobing@hit.edu.cn ) \\ Harbin Institute of Technology \\ Qingliang Zhao \\ Harbin Institute of Technology, \\ T.S. Mahmoud \\ Benha University
}

\section{Research Article}

Keywords: Grinding, Patterned grinding wheels, Structured surface, Modeling

Posted Date: June 17th, 2021

DOI: https://doi.org/10.21203/rs.3.rs-503465/v1

License: (a) (1) This work is licensed under a Creative Commons Attribution 4.0 International License. Read Full License

Version of Record: A version of this preprint was published at The International Journal of Advanced Manufacturing Technology on January 6th, 2022. See the published version at https://doi.org/10.1007/s00170-021-08296-5. 


\title{
Modeling and simulation of the advanced structured surfaces machined by specially patterned grinding wheels via the structuring grinding process
}

\author{
Amr Monier ${ }^{1}$ - Bing Guo*1 . Qingliang Zhao*1 . T. S. \\ Mahmoud $^{2}$. \\ [1] Center for Precision Engineering, School of Mechatronics Engineering, Harbin \\ Institute of Technology, Harbin, 150001, China \\ [2] Mechanical Engineering Department, Shoubra Faculty of Engineering, Benha \\ University, Cairo, 11629, Egypt
}

\begin{abstract}
Author: Amr Monier
E-mail: amr.monier@hit.edu.cn Telephone :

0086-18845871045

Address: P.O. Box 413, Harbin Institute of Technology,

Harbin, 150001, China .
\end{abstract}

Corresponding author: Bing Guo

E-mail: guobing@hit.edu.cn Telephone :

0086-13654501138.

Corresponding author: Qingliang Zhao

E-mail: zhaoqingliang@hit.edu.cn Telephone :

0086-13674680330 .

Author: T. S. Mahmoud

E-mail: tamer.abdelmagid@feng.bu.edu.eg Telephone :

002-01220604020 


\begin{abstract}
In this work, the ability to reshape the grinding wheels with special patterns to produce advanced structured surfaces with several geometries is studied. Firstly, a mathematical model is built for the process relating geometries of the grinding wheel, geometries of wheel patterns, the produced structured surface with the grinding operating parameters. Then, different regular and irregular geometries are designed to be patterned over the wheel surface. Afterward, a simulation method to express the patterned wheels and the structured surface at different working conditions is developed. The effects of the pattern geometries on the obtained structured surfaces are investigated.
\end{abstract}

Keywords Grinding · Patterned grinding wheels $\cdot$ Structured surface $\cdot$ Modeling

\section{Introduction}

The increasing developments in the fields of modern industries and advanced manufacturing applications require producing surfaces with a determined function performance able to satisfy specific requirements. One frequent solution that has been recently applied is represented in the creation of the structured surfaces of parametric characteristics $[1,2,3]$. Through the use of the surfaces with structures, quantitative relationships can be established between the structural characteristics and the required functional performance of the surfaces. Surface structures should have characteristic parameters that can be designed according to both the selected manufacturing metho and the demanded functions for engineering applications. In recent researches, it has been proved that surface structures improve effectively several areas in tribology such as friction, wear, lubrication, and energy consumption $[4,5,6]$. Hence, the research on the manufacturing of controlled propriety surface structures on engineering surfaces is of great significance [7]. The significance of structured surfaces in modern applications can be found in several areas such as electronics [8], optics[9], and health [10].

Different methods can be used to fabricate the surface structures [11, 12, 13]. Recently, the grinding method has been used for machining surface structures in macro/micro-scale using what is called structured grinding wheels $[14,15]$. This method is distinguished by the high efficiency, simplicity, and low cost. It produces different forms of surface structures repetitively without the need for additional manufacturing steps saving additional cost and time. Different studies have investigated the effect of using the structured/patterned grinding wheels on the overall process efficiency[16, 17]. To use the grinding process for machining a structured ground surface, the selected pattern should be formed on the wheel surface itself and subsequently transferred to the workpiece during processing operations. Modeling of the structuring grinding process to relate the properties of the final structure to the different operating parameters of the grinding method can be used for improving and optimizing the overall process efficiency.

Several studies used the grinding method to model and produce surface structures using the structured grinding wheel, but with some limitations on the obtained structured surfaces. Stpien $[18,19]$ used deep-dressed single/doublehelical structured wheels to produce the three basic shapes of surface structures in one or two grinding paths with selected grinding conditions. Denkena et al. [20] enhanced the performance of turbine blades by generating riblet structures fabricated through the grindding method. Silva et al [21] and Oliveira et al. [22] presented different dressing and structuring methods that enabled forming pre-configurable patterns for the surface structures using grinding. Mohamed et al. [23] utilized the modeling method developed by Liu et al. [24] to predict the produced surface structures using the circumferentially structured grinding wheels.

The geometry of surface structures is one of the main characteristics that can be used for defining the properties of surface structures. 


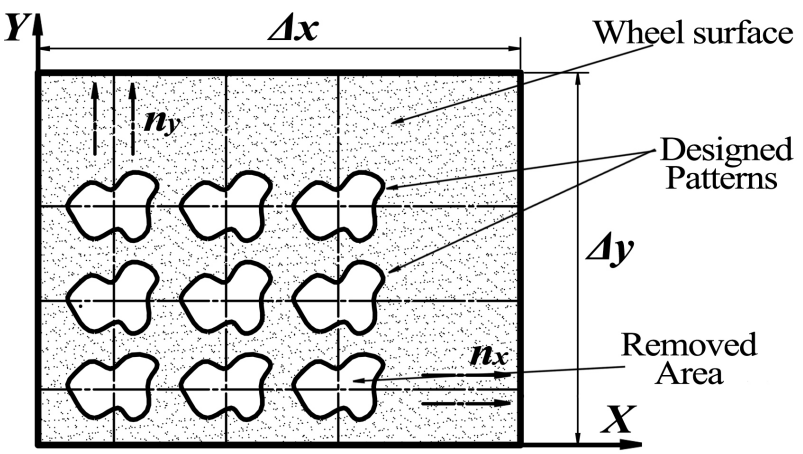

(a)

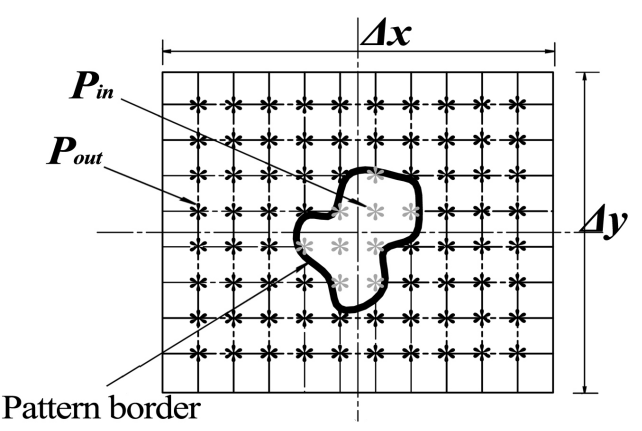

(b)

Fig. 1: (a) The designed unit segment of the grinding wheel in plain view, (b) defining of the point position over the unit segment.

Machining the surface structures in a repetitive way with different sizes controlled through the grinding parameters characterize the used of the grinding method. However, the majority of the work found in the literature focused on the straight/helical patterns for the wheel surface and the related structures on the workpiece surfaces. Studying the ability of the grinding wheels to be patterned in other geometries and the related effects on the obtained structured surfaces have not been studied before. This work is aiming to model and simulate the patterned grinding wheels with new pattern geometries and study the effects of these geometries on the related structured surfaces.

Therefore, in this work, the ability for patterning the grinding wheel with new regular and irregular patterns is presented. The effects of the patterned wheels on the related structured surfaces at different working conditions are investigated. A mathematical model for the process relating the geometries of the patterned wheel and the structured surface with the selected grinding condition is built. Different regular and irregular patterns are defined and applied on the grinding wheels. A simulation method for the process using the programming languages is examined. Different parameters affecting the surface structuring process have been explained.

\section{Modeling of grinding operations for the structured surfaces with special patterned grinding wheels}

To create the model of the patterned grinding wheel, a unit segment for the grinding wheel surface is designed according to the pattern geometry required to be shaped on the wheel surface. Fig. 1 explains the principles for building the model of the patterned wheel. In Fig. 1a, a $2 \mathrm{D}$ sketch of the unit segment of the grinding wheel with $n_{x}$ by $n_{y}$ number of patterns positioned at determined locations is designed according to the required special pattern geometry. The geometrical pair $(\Delta x \& \Delta y)$ represent the dimensions of the unit segment along the $x$-axis and $y$-axis respectively, while each wheel pattern has an area $\left(a_{s}\right)$. The proposed model assumes that the fraction of remained area $\left(A_{r}\right)$ of the unit segment is defined as structuring ratio $\left(\gamma_{s}\right)$, such that:

$\gamma_{s}=\frac{A_{r}}{A_{t}}$

Where $\left(A_{t}\right)$ is the to total area of the unit segment. Then, the total patterned area $\left(A_{p}\right)$ of the unit segment can be determined as the following:

$A_{p}=A_{t} \cdot\left(1-\gamma_{s}\right)$ 


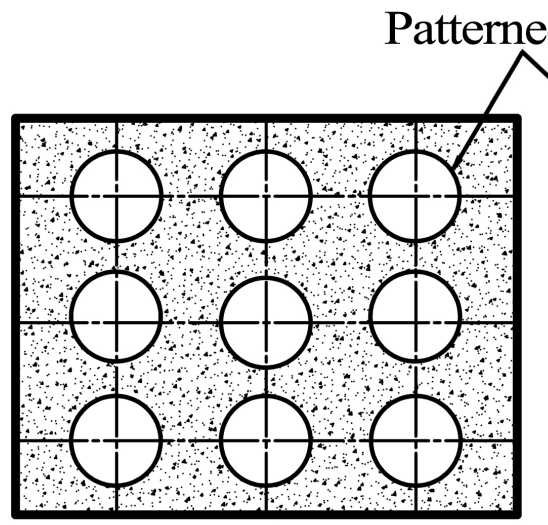

(a)

Patterned area

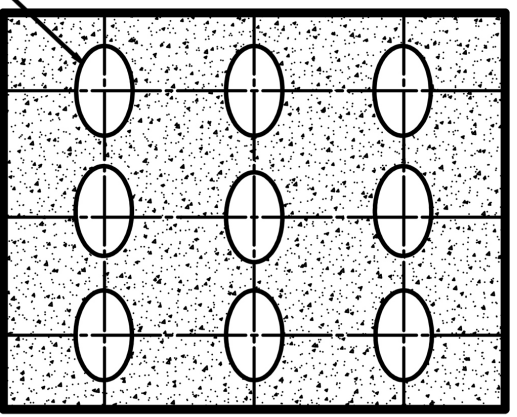

(b)

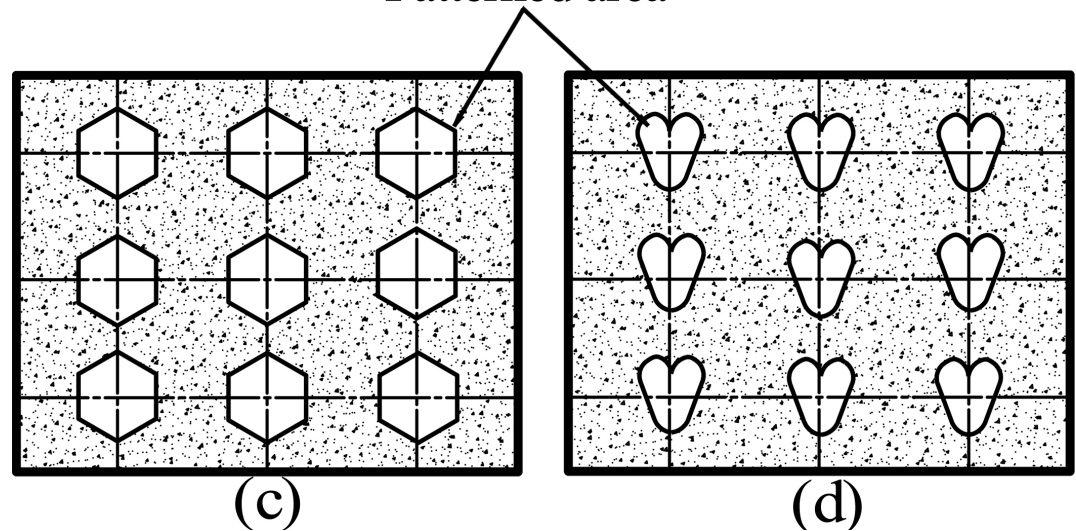

Fig. 2: The different geometries for the wheel patterns, (a) the circular patterns, (b) the elliptical patterns, (c) the polygonal patterns, and (d) the heart-like patterns.

Assuming a uniform area for all patterns, Therefore:

$a_{s}=\frac{A_{p}}{n_{x} \cdot n_{x}}$

Then, the geometry of the unit segment of the structured wheel is converted to be a net and determined as an array of points in $x-y$ coordinates. See Fig. 1b. Assuming $c_{x i}$ and $c_{y i}$ are the center coordinates of the pattern area $a_{i}$, the model selects the optimum positions to place the patterns over the designed area of the unit segment. Afterward, the coordinates of all points either inside or outside the wheel pattern area are allowed to be identified by calculating mathematically the borders of each pattern area as follows:

$f(x, y)=\left\{\begin{array}{l}f\left(x_{i}\right), 1 \leq i \leq n \\ f\left(y_{i}\right), 1 \leq i \leq n\end{array}\right.$

Where $x_{i}$ and $y_{i}$ are the coordinates of the $n$ points of the unit segment.

Fig. 2 shows different geometries that can be patterned on the grinding wheel surface. If the wheels patterns have circular geometries, the borders can be determined using basic mathematics as follows [25]:

$\left(x_{i}-c_{x i}\right)^{2}+\left(y-c_{y i}\right)^{2}=r^{2}$

Where $x_{i}$ and $y_{i}$ are the coordinates of any point in the $x-y$ plane and $r_{c}$ is the radius of the wheel pattern. For polygonal patterns, the 
side length $L_{\text {side }}$ can be related to the circumscribed circle radius $r_{c}$ by:

$L_{\text {side }}=2 \cdot r_{c} \cdot \sin \left(\frac{\pi}{N_{\text {side }}}\right)$

Where $N_{\text {side }}$ represents the number of sides for the designed polygon.

For elliptical geometries:

$\frac{x_{i}^{2}}{a^{2}}+\frac{y_{i}^{2}}{b^{2}}=1$

Where $a$ and $b$ are the major and minor axes half lengths of the elliptical pattern.

Moreover, irregular geometries such as heartlike shapes can also be modeled by controlling the driving formula for their $x_{i}$ and $y_{i}$ coordinates. For example, for heart-like geometries:

$$
\begin{aligned}
x_{i}=a \cdot \sin (\theta) \\
y_{i}=b \cos (\theta)-c \cos (2 \theta)-d \cos (3 \theta)- \\
\quad e \cos (\theta)-f \cos (14 \theta)
\end{aligned}
$$

Where $a, b, c, d, e$, and $f$ are constants.

After calculating the coordinates for all points over the unit segment in the $x-y$ plane, points coordinates in z-direction represent the depth of the points on the unit segment. The depth is specified according to the position of its point on the unit segment area. If the patterns structured on the wheel surface have depth $d_{s}$, then the points inside the pattern border are supposed to be with $d_{s}$ values. Otherwise, the $z$-coordinates become extinct. So, mathematically:

$z_{i}= \begin{cases}0 & P_{i}=P_{\text {out }} \\ d_{s} & P_{i}=P_{\text {in }}\end{cases}$

Where $P_{\text {in }}$ and $P_{\text {out }}$ represent the locations either inside or outside the pattern border respectively.

After that, the points coordinates in the $x$, $y$, and $z$ directions are converted into the polar system and the unit segment of the grinding wheel is created. See Fig. 3a. Then, the unit segment is repeated in the circumferential and radial directions to create the completed design of the patterned wheel shown in Fig. 3b.

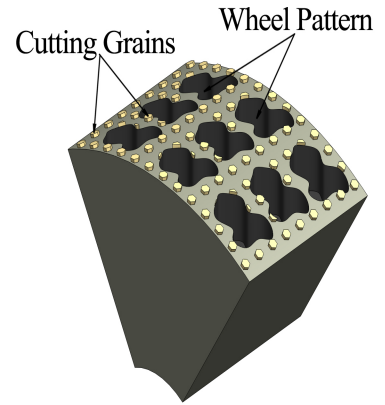

(a)

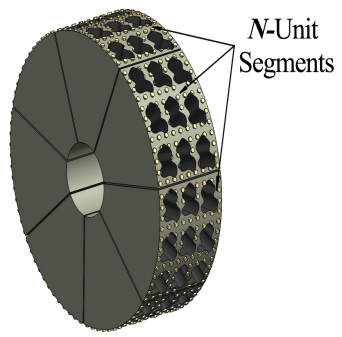

(b)
Fig. 3: (a) The unit segment in polar coordinates and isometric view, (b) repeating the unit segment $N$ times to build the grinding wheel.
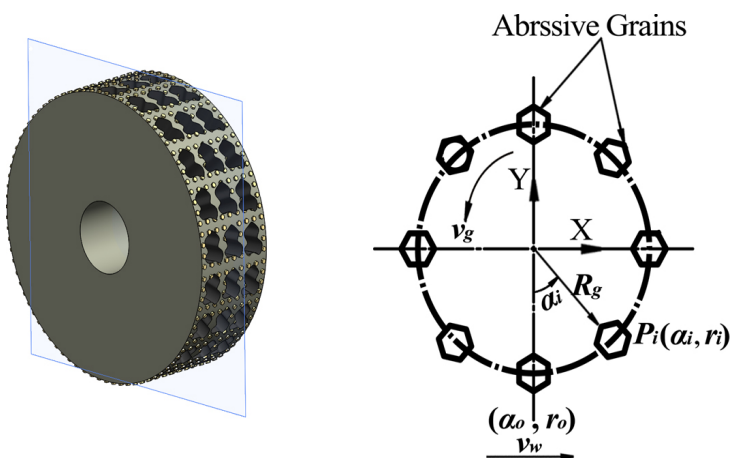

Fig. 4: The kinematic model of the patterned grinding wheel.

For simplicity, the unit segment is assumed to be repeated in one direction (circumferential direction). The circumferential distance from one point on a unit segment to the matching one on the next segment is titled circular pitch $P_{c}$. Assuming the grinding wheel has a radius $\left(R_{g}\right)$, the number of the unit segments that should be repeated to create the complete shape of the grinding wheel can be determined as:

$N=\frac{2 \pi \cdot R_{g}}{P_{c}}$

Fig. 4 illustrates the way followed to create the kinematic model for the patterned wheel. The wheel is divided into several sections cut across the wheel's longitudinal axis. Each one 
is assumed to have a total number of points $P_{n}$ represents the number of abrasive cutting grains. An abrasive point $P_{i}$ is positioned at an angle $\left(\alpha_{i}\right)$ and radius $r\left(\alpha_{i}\right)$. The polar position of the point $\left(P_{i}\right)$ is determined by the pair of parameters $\left(\left(\alpha_{i}\right) \& r\left(\alpha_{i}\right)\right)$ respectively. The angle $\left(\varphi_{i}\right)$ determines the orientation of the point $P_{i}$ from its home position after starting up the grinding operation. Using Equation 10 , the radial position of the point $\left(P_{i}\right)$ is calculated throughout subtracting the depth $(d s)$ from the wheel radius $\left(R_{g}\right)$ and substituting in Eq.10 for $z_{i}\left(P_{i}\right)$ by $d_{s}\left(P_{i}\right)$, therefore:

$R_{g}= \begin{cases}R_{g}, & P_{i}=P_{\text {out }} \\ R_{g}-d_{s}, & P_{i}=P_{\text {in }}\end{cases}$

After determining the polar position of all points around the wheel $2 \mathrm{D}$ section, all sections points are similarly specified and a complete definition of the wheels kinematic model is obtained.

Fig. 5 explains the kinematics of the grinding operation proposed in this work to transfer the wheel patterns to the workpiece surface as structures. The grinding wheel and the workpiece operate at working velocities $v_{g}$ and $v_{w}$ respectively, while the grinding interaction between them is executed at $\left(d_{c}\right)$ depth of cut . To get the structured surface, the presented model assumed that the grinding wheel rolls over the workpiece surface upon starting the grinding process as a result of the translation and the rotation motions in a perfect cycloidal path.

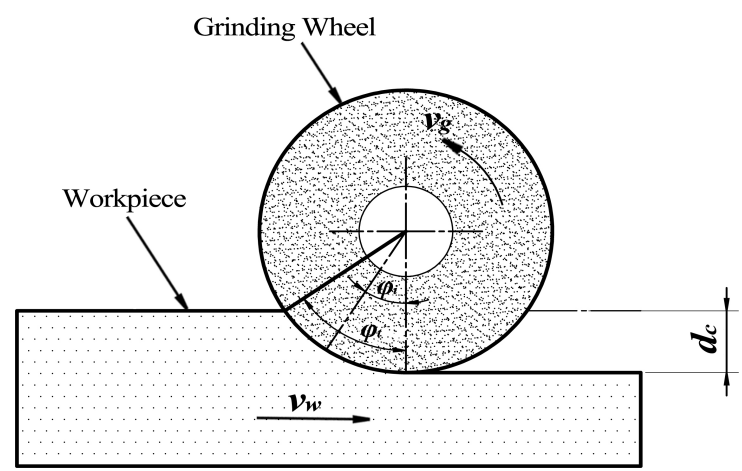

Fig. 5: A sketch of the kinematics of the grinding operation.
The structures over the workpiece surface are produced in such a way similar to that followed to build up the structured grinding wheel. The patterns shaped on each unit segment of the wheel surface are reflected on the workpiece surface to a specific scale while the grinding wheel is rolling over the ground surface. As illustrated in Fig. 6, the linear distance on the workpiece surface between the engaging positions of two successive unit elements with the workpiece surface is named longitudinal pitch $P$. Accordingly, the circular pitch of the grinding wheel $\left(P_{c}\right)$ is related to the longitudinal pitch $(P)$ of workpieces structures as follows:

$P=P_{c} \cdot \frac{v_{\mathrm{w}}}{v_{\mathrm{g}}}=P_{c} \cdot v^{*}$

And $v^{*}$ is the ratio between the operating velocities of the workpiece and the grinding wheel respectively.

The geometry of each structure on the workpiece surface is defined by structure width $\left(S_{w}\right)$ and structure height $\left(S_{h}\right)$. See Fig. 6 . The structure width $\left(S_{w}\right)$ is the max-width of the workpiece surface pattern in the direction parallel to the grinding direction. Similarly, the structure height $\left(S_{h}\right)$ is the max height of the pattern over the workpiece ground surface. The values of the geometrical pair $\left(S_{w} \& S_{h}\right)$ in addition to the longitudinal pitch $(P)$ are controlled by the of the grinding wheel geometry, the designed pattern geometry, and the operating parameters of the grinding process.

Fig. 7 explains the effect of eccentricity between the patterns on the unit segment on the structured workpiece. In Fig. 7, the helical angle $(\psi)$ defines the orientation of the distance between centers of wheel patterns from its longitudinal axis. The angle $(\vartheta)$ represents the orientation of the centroids of the structures machined on the workpiece surface. According to the geometry shown in Fig. 7:

$\tan \vartheta=\frac{P}{P_{c}} \cdot \tan \psi$

Using Eq. (13), then:

$\tan \vartheta=\frac{v_{w}}{v_{g}} \cdot \tan \psi$ 


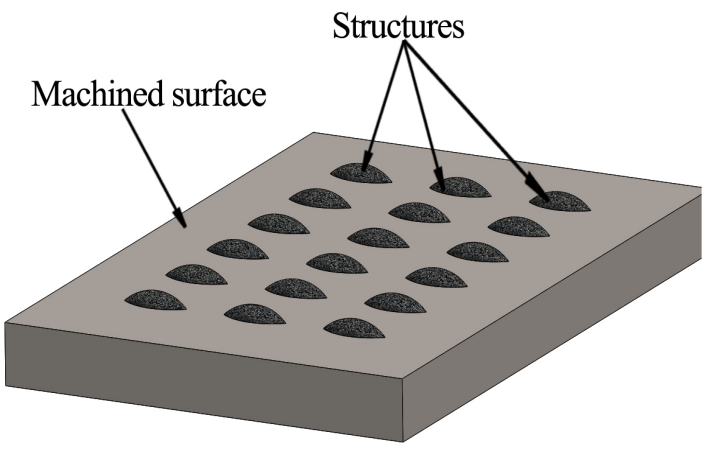

(a)

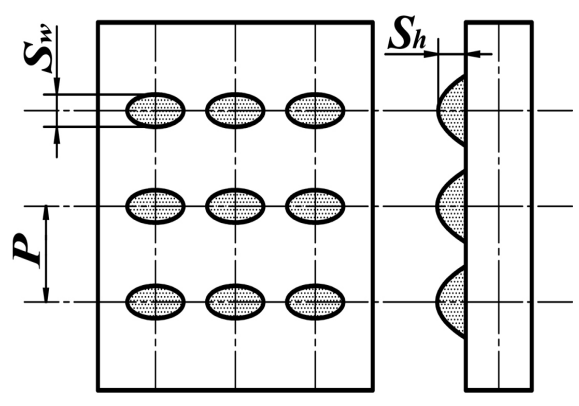

(b)

Fig. 6: (a) A schematic view of the structured surface, (b) A plan view of the structured surface with its geometrical parameters.

Then, in the case of up-grinding:

$\vartheta=\arctan \left[\frac{v_{w}}{v_{g}} \cdot \tan \psi\right]$

In down-grinding:

$\vartheta=\pi-\arctan \left[\frac{v_{w}}{v_{g}} \cdot \tan \psi\right]$

When the structuring process starts up, the cycloidal path of any abrasive point on the grinding wheel and the scaled profile reflected on the workpiece surface can be supposed according to the time consumed during the processing. The exhausted time $t_{i}$ by the abrasive grain of number, $P_{i}$ to alternate its home position from $\left(\alpha_{i}\right)$ to $\left(\varphi_{i}\right)$ can be supposed as the

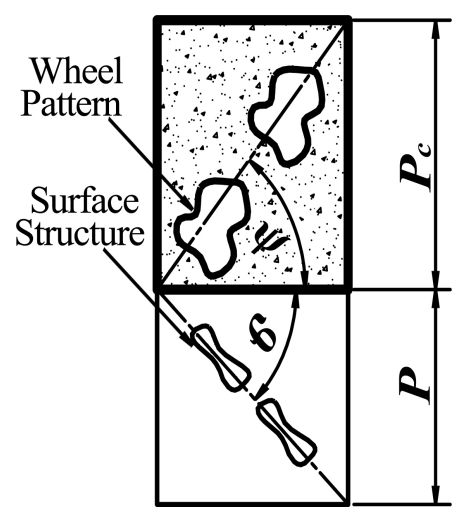

Fig. 7: The relationship of the helical angle of wheel patterns with the slope angle of surface structures. following:

$t_{i}=\frac{r_{i}}{v_{g}} \cdot\left(\varphi_{i}-\alpha_{i}\right)$

Then, the path coordinates in the feeding direction $\left(X_{i}\right)$ and the direction normal to it $\left(Y_{i}\right)$ defined by:

$\left\{\begin{array}{c}\mathrm{X}_{\mathrm{i}}(t)=v_{w} \cdot t_{i}+r_{i} \cdot \sin \varphi_{i} \\ \mathrm{Y}_{\mathrm{i}}(t)=R_{g}-d_{g}-r_{i} \cdot \cos \varphi_{i}\end{array}\right.$

Using Eq. (18) \& Eq. (19), in the case of upgrinding:

$\left\{\begin{array}{c}\mathrm{X}_{\mathrm{i}}(t)=v_{w} \cdot t_{i}+r_{i} \cdot \sin \left(\left(\frac{v_{g}}{r_{i}} \cdot t_{i}\right)+\alpha_{i}\right) \\ \mathrm{Y}_{\mathrm{i}}(t)=R_{g}-d_{g}-r_{i} \cdot \cos \left(\left(\frac{v_{g}}{r_{i}} \cdot t_{i}\right)+\alpha_{i}\right)\end{array}\right.$

In down-grinding:

$\left\{\begin{array}{l}\mathrm{X}_{\mathrm{i}}(t)=-v_{w} \cdot t_{i}+r_{i} \cdot \sin \left(\left(\frac{v_{g}}{r_{i}} \cdot t_{i}\right)+\alpha_{i}\right) \\ \mathrm{Y}_{\mathrm{i}}(t)=R_{g}-d_{g}-r_{i} \cdot \cos \left(\left(\frac{v_{g}}{r_{i}} \cdot t_{i}\right)+\alpha_{i}\right)\end{array}\right.$

\section{Simulation Results and Discussion}

Simulation of the structuring grinding processes using programming languages is an attractive way for estimating both the designed patterned 
Table 1: The proposed parameters for the wheel geometry and the grinding process with the circular patterned wheels.

\begin{tabular}{c|c|c|c}
\hline Wheel Radius $\left(R_{g}\right) \mathrm{mm}$ & structuring ratio $\left(\gamma_{s}\right)$ & Depth of cut $d_{c}$ & Velocity Ratio $v^{*}$ \\
\hline 15 & 0.5 & 0.01 & 0.10 \\
\hline
\end{tabular}

Table 2: The designed geometrical parameters for the circular patterns of the grinding wheel. Circular Pattern No.

\begin{tabular}{c|c|c|c|c|c|c|c|c}
\hline \multicolumn{3}{c|}{$\mathrm{a}$} & \multicolumn{3}{c|}{$\mathrm{b}$} & \multicolumn{3}{c}{$\mathrm{c}$} \\
\hline$r(m m)$ & $d_{s}(\mathrm{~mm})$ & $\psi(\operatorname{deg})$ & $r(\mathrm{~mm})$ & $d_{s}(\mathrm{~mm})$ & $\psi(\mathrm{deg})$ & $r(\mathrm{~mm})$ & $d_{s}(\mathrm{~mm})$ & $\psi(\operatorname{deg})$ \\
\hline 0.5 & 0.5 & 0 & 0.65 & 0.65 & 30 & 0.80 & 0.8 & 45 \\
\hline
\end{tabular}

wheels and the obtained structured surfaces at different operating parameters for the grinding process. The simulation process depends on the geometry of the grinding wheel, the geometry of the wheel pattern, and the working parameters of the grinding operation. The geometry of the grinding wheel is defined by the wheel radius $\left(R_{g}\right)$ and the structuring ratio $\left(\gamma_{s}\right)$. The geometry of the wheel pattern will be defined later in the following sections. The working of the grinding process includes the working velocities $\left(v_{g} \& v_{w}\right)$ and the grinding depth of cut $\left(d_{c}\right)$. In this study, the wheel geometry and the grinding parameters are designed to be constant while the major changes happen to the patterns geometrical parameter. This is aiming to clarify the effects of the patterns geometrical parameters on the produced surface structures with being affected neither by the wheel geometry nor the grinding parameters.

On the other side, the geometry of the structured surfaces resulted from wheels with different patterns and at determined grinding conditions will be simulated and evaluated. The geometry of the structures produced on the workpiece surface is determined by the values of the structure width $\left(S_{w}\right)$, the structure height $\left(S_{h}\right)$, the longitudinal pitch $(P)$, and the slope angle $(\vartheta)$. The ability of the presented model to estimate the structured surfaces produced by the designed wheels of regular and irregular patterns mentioned in the previous section will be measured. This is illustrated in the subsequent sections.
3.1 Simulation of the structured surfaces produced by structured wheels with round patterns

The first pattern presented for the grinding wheels is the wheels with the round patterns. This includes wheels with circular patterns and elliptical patterns. For the circular patterns, the pattern geometry is defined by the radius of the circular pattern $(r)$. For the elliptical patterns, half lengths of the major and minor axes $(a \& b)$ control the pattern geometry. Also, the pattern geometry includes the required helical angle $(\psi)$ and the depth $\left(d_{s}\right)$ of the patterns produced on the wheel surface. Table 1 includes the geometrical parameters of the grinding wheel and the selected grinding condition used in this study. Table 2 contains different designs for the circular patterns required to be shaped on the grinding wheel surface.

Fig. 8 shows the unit segments of the circular patterns mentioned in Table 2, the grinding wheels, and the related structured surfaces with $2 \mathrm{D}$ profiles across the unit lengths of structures. As shown in Fig. 8, when the radius $(r)$ of the wheel pattern increases, for the same area of the unit segment, the number of patterns on the wheel surface decreases. Besides, the peripheral distance between the successive patterns increases. In this figure, the difference in the number of patterns, the pattern depths, and the helical angle between centers can be noted.

For the side of the structured surfaces, it can be shown that as the pattern radius $(r)$ 


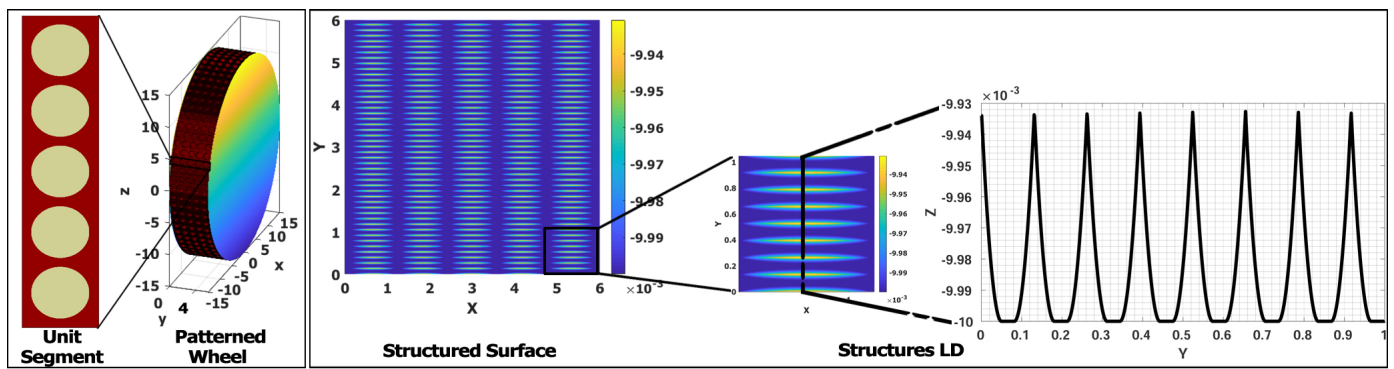

(a)
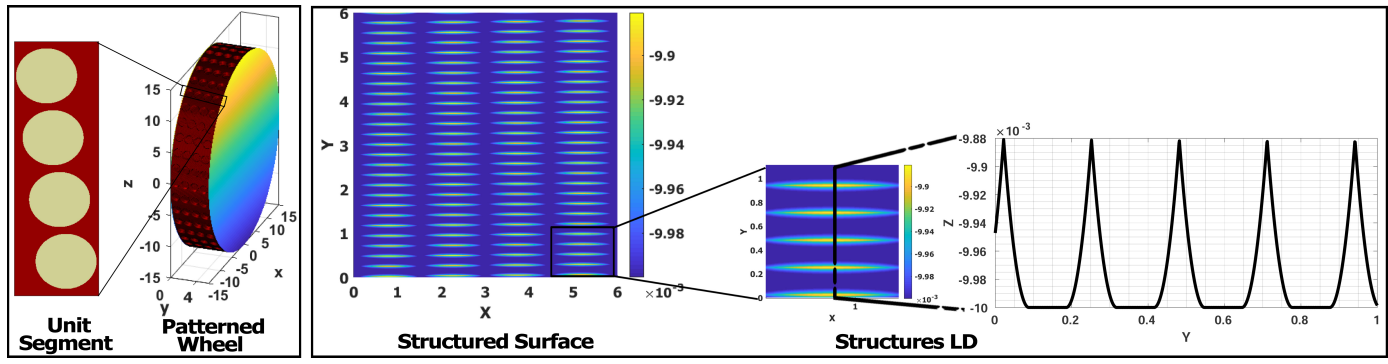

(b)
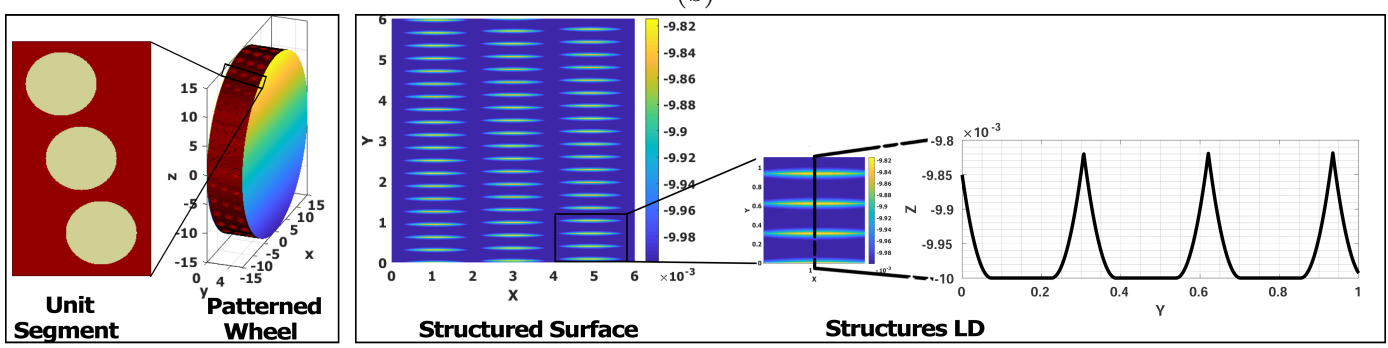

(c)

Fig. 8: The grinding wheels and the related structured surfaces with different geometries of the circular patterns.

Table 3: The geometrical parameters of the structured surfaces obtained by the circular patterned wheels

\begin{tabular}{|c|c|c|c|c|c|c|c|c|c|c|c|}
\hline \multicolumn{12}{|c|}{ The structured surface of circular pattern $\mathrm{No}$. } \\
\hline \multicolumn{4}{|c|}{$\mathrm{a}$} & \multicolumn{4}{|c|}{$\mathrm{b}$} & \multicolumn{4}{|c|}{$\mathrm{c}$} \\
\hline$S_{w}(\mathrm{~mm})$ & $P(\mathrm{~mm})$ & $\vartheta(\operatorname{deg})$ & $S_{h}(\mu m)$ & $S_{w}(\mathrm{~mm})$ & $P(\mathrm{~mm})$ & $\vartheta(\operatorname{deg})$ & $S_{h}(\mu m)$ & $S_{w}(\mathrm{~mm})$ & $P(\mathrm{~mm})$ & $\vartheta(\operatorname{deg})$ & $S_{h}(\mu m)$ \\
\hline 0.085 & 0.131 & 0 & 0.070 & 0.112 & 0.234 & 3.31 & 0.120 & 0.129 & 0.300 & 5.71 & 0.182 \\
\hline
\end{tabular}

increases, the structure width $\left(S_{w}\right)$, the structure height $\left(S_{h}\right)$, and the longitudinal pitches $(P)$ between surface structures increase. See Fig. 8 and Table 3 . The reason is that with the increasing pattern radius $(r)$, the circumferential distance between wheel patterns increases with decreasing the number of patterns over the wheel surface. If the circumferential distance between centers of two successive pat- terns is considered as the circular pitch $\left(P_{c}\right)$ of the patterned wheel. Therefore, the circular pitch $\left(P_{c}\right)$ of the patterned wheel and the related longitudinal pitch $(P)$ on the workpiece surface increase with the increasing pattern radius $(r)$. Hence, the structure's width $\left(S_{w}\right)$ increases with the increasing longitudinal pitch $(P)$ allowing the structure's height $\left(S_{h}\right)$ to acquire a higher value. 

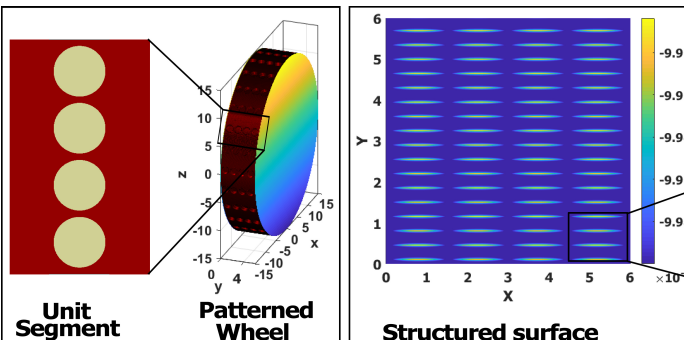

Structured surface

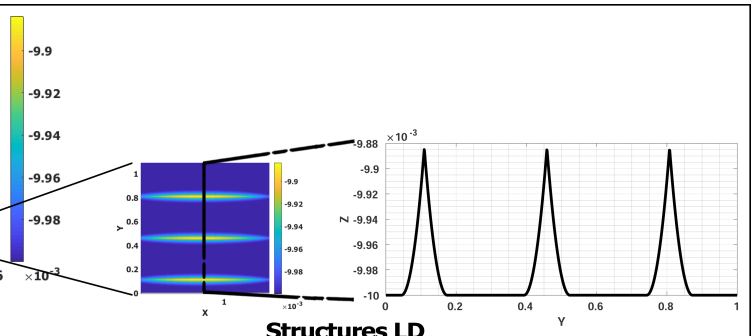

(a)

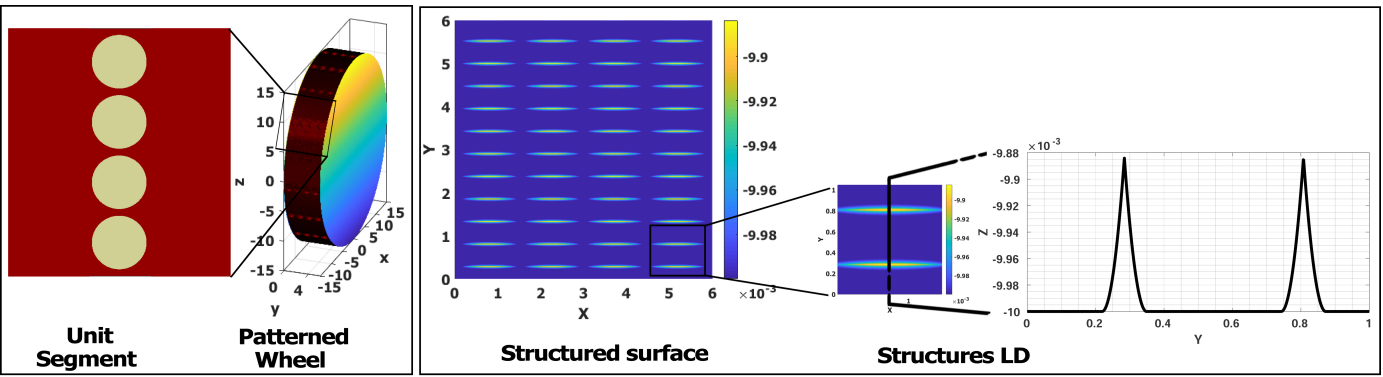

(b)
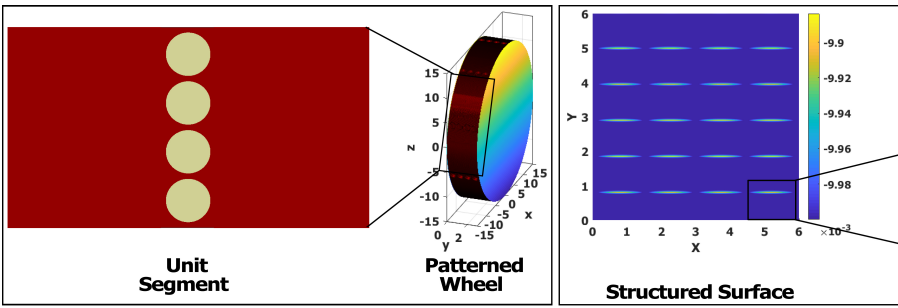

(c)

Fig. 9: The circular patterned wheels and the related structured surfaces at the increasing structuring ratio.

Moreover, assuming the number of the workpiece surface structures per unit length is defined as structures linear density (structures $L D)$. Therefore, the structures $L D$ decreases with the increasing pattern radius $(r)$. This can be explained as the rate of the passing patterns over the workpiece surface decrease, for the same grinding condition, with the increasing pattern radius $(r)$. The changing in the the structures $L D$ with the increasing pattern radius can be shown clearly in Fig. 8 () where the $2 \mathrm{D}$ profiles are taken across the unit length of surface structures. To increase the structures $L D$ at a raising value of the pattern radius $(r)$, the velocity ratio should be modified.
This previous explanation is agreed with the modeling principles explained and formulated in Section 2. Table 3 includes the geometrical parameters for the structured surfaces illustrated in Fig. 8.

The structuring ratio $\left(\gamma_{s}\right)$ of the patterned grinding wheels can also control the degree of surface structuring. Fig. 9 shows different designs for the grinding wheel of the circular pattern of number (b) in Table 2 and the related structured surfaces but at increasing value for the structuring ratio $\left(\gamma_{s}\right)$ and at a zero helical angle $(\psi=0)$. Comparing the unit segments, patterned wheels, and structured surfaces in Fig. 9 with that shown in Fig. 8b. It is clear that as the structuring ratio $\left(\gamma_{s}\right)$ increases, the 
Table 4: The proposed parameters for the wheel geometry and the grinding process with the elliptical patterned wheels

\begin{tabular}{c|c|c|c}
\hline Wheel Radius $\left(R_{g}\right) \mathrm{mm}$ & structuring ratio $\left(\gamma_{s}\right)$ & Depth of cut $d_{c}$ & Velocity Ratio $v^{*}$ \\
\hline 20 & 0.73 & 0.01 & 0.128 \\
\hline
\end{tabular}

Table 5: The designed geometrical parameters for the elliptical patterns of the grinding wheel

\begin{tabular}{|c|c|c|c|c|c|c|c|c|c|c|c|}
\hline \multicolumn{12}{|c|}{ Elliptical Pattern $N o$. } \\
\hline & & & & & & & & & & & \\
\hline$a(\mathrm{~mm})$ & $b(\mathrm{~mm})$ & $d_{s}(\mathrm{~mm})$ & $\psi(\mathrm{deg})$ & $a(\mathrm{~mm})$ & $b(\mathrm{~mm})$ & $d_{s}(m m)$ & $\psi(d e g)$ & $a(\mathrm{~mm}) b$ & $(\mathrm{~mm})$ & $d_{s}(\mathrm{~mm})$ & $\psi(\operatorname{deg})$ \\
\hline 0.50 & 0.25 & 0.5 & 0 & 0.65 & 0.325 & 0.65 & 30 & 0.80 & 0.40 & 0.8 & 45 \\
\hline
\end{tabular}

Table 6: The geometrical parameters of the structured surfaces obtained by the elliptical patterned wheels

\begin{tabular}{c|c|c|c|c|c|c|c|c|c|c|c}
\hline \multicolumn{10}{c}{ The structured surface of elliptical pattern No. } \\
\hline \multicolumn{9}{c|}{ a } & \multicolumn{9}{c|}{ b } & \multicolumn{4}{c}{ c } \\
\hline$S_{w}(\mathrm{~mm})$ & $P(\mathrm{~mm})$ & $\vartheta(\mathrm{deg})$ & $S_{h}(\mu \mathrm{m})$ & $S_{w}(\mathrm{~mm})$ & $P(\mathrm{~mm})$ & $\vartheta(\mathrm{deg})$ & $S_{h}(\mu \mathrm{m})$ & $S_{w}(\mathrm{~mm})$ & $P(\mathrm{~mm})$ & $\vartheta(\mathrm{deg})$ & $S_{h}(\mu \mathrm{m})$ \\
\hline 0.097 & 0.152 & 0 & 0.0758 & 0.133 & 0.274 & 4.23 & 0.130 & 0.174 & 0.387 & 7.30 & 0.200 \\
\hline
\end{tabular}

total number of patterns on the wheel surface decreases. As the increasing of the structuring ratio means decreasing the total patterned area of the grinding wheel. See Eq. (1). Therefore, the circular pitch $\left(P_{c}\right)$ and the related longitudinal pitch $(P)$ increase, and the structures $L D$ decreases with the increasing structuring ratio $\left(\gamma_{s}\right)$. See Fig. 9. However, the structure width $\left(S_{w}\right)$ and the structure height $\left(S_{h}\right)$ of the workpiece surface structures have not changed from that shown in Fig. 8b as the patterns geometry and the operating parameters are similar.

For the elliptical patterns, the selected operating parameters for the wheel geometry and the grinding process are found in Table 4. The designed geometries for the elliptical patterns are declared in Table 5. Fig. 10 illustrates the elliptical patterned wheels and the related structured surfaces obtained using the working condition stated in Table 4. Similarly, with the increasing pattern size, the number of patterns on the wheel surface decreases, and accordingly the peripheral distance between them increases. As a result, the size of structures produced on the workpiece surface increases, and the structures $L D$ decreases. Table 6 contains the geometrical parameters of the structured surfaces represented in Fig. 10.

Also, in Fig. 11, an example for the patterned wheel of number (b) in Table 5 (at zero helical angle $(\psi))$ and the related structured surfaces at an increasing structuring ratio $\left(\gamma_{s}\right)$ is figured out. The behavior of the structured surfaces with the increasing structuring ratio $\left(\gamma_{s}\right)$ is similar to that of the circular patterned wheel. The geometry of the structure itself is the same for all patterned wheels similar to that shown in Fig. 10b but the longitudinal pitches $(P)$ between them increase due to the increasing circular pitch $\left(P_{c}\right)$ with the increasing structuring ratio $\left(\gamma_{s}\right)$.

3.2 Simulation of the structured surfaces produced by structured wheels with polygonal patterns

Next, the structured surfaces produced by grinding wheels of polygonal patterns are investigated. In this study, the geometry of the polygonal pattern is defined by the circumscribed circle radius $\left(r_{c}\right)$ and the number of the polygons sides $\left(N_{\text {side }}\right)$. The operating conditions used in this investigation for the geometry of the grinding wheel and the grinding process are listed in Table 7 . Table 8 includes the geometries of the polygonal patterns required to be shaped on the grinding wheel surface. Three types of polygonal patterns have been tried with different geometrical parameters. This includes square, hexagonal, and octagonal patterns with increasing values of the circumscribed 

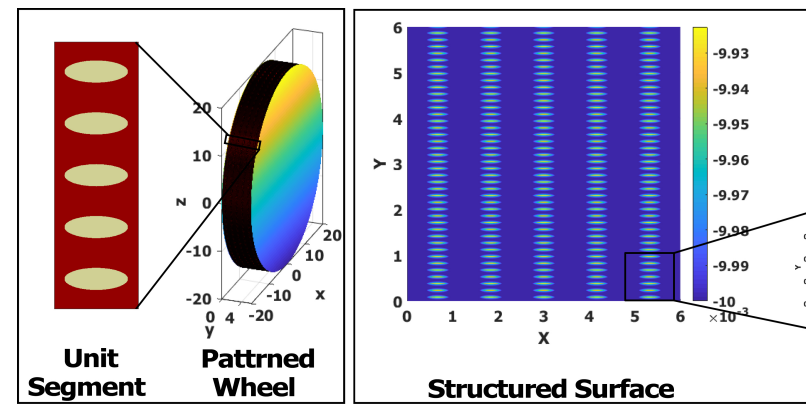

Structured Surface

(a)
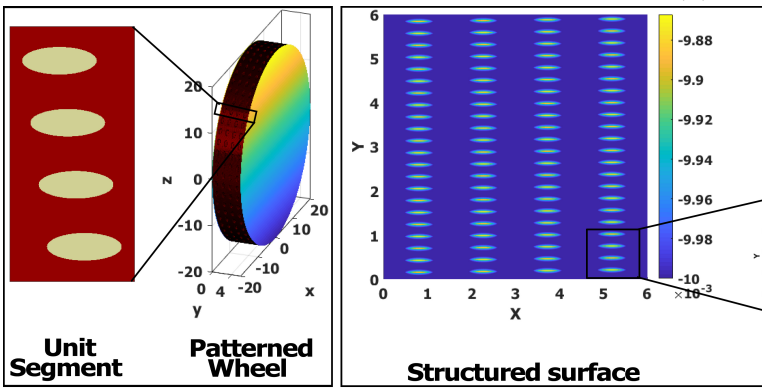

Structured surface

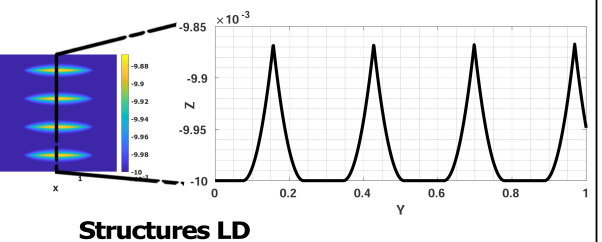

(b)
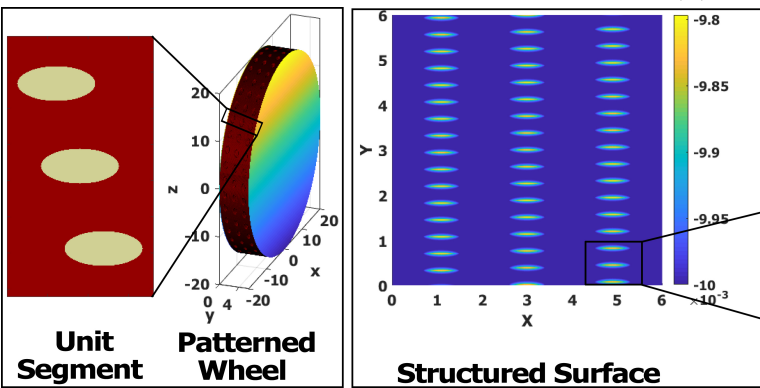

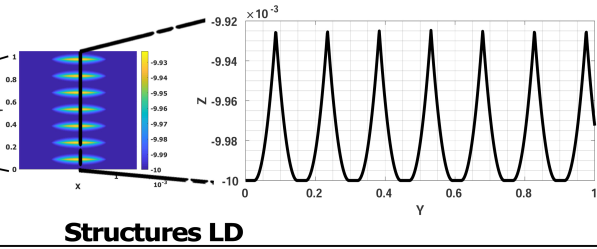

\section{(c)}

Fig. 10: The grinding wheels and the related structured surfaces with different geometries for the elliptical patterns.

Table 7: The proposed parameters for the wheel geometry and the grinding process with the polygonal patterned wheels

\begin{tabular}{c|c|c|c}
\hline Wheel Radius $\left(R_{g}\right) m m$ & structuring ratio $\left(\gamma_{s}\right)$ & Depth of cut $d_{c}$ & Velocity Ratio $v^{*}$ \\
\hline 15 & 0.65 & 0.01 & 0.167 \\
\hline
\end{tabular}

Table 8: The designed geometrical parameters for the polygonal patterns of the grinding wheel

\begin{tabular}{c|c|c|c|c|c|c|c|c|c|c|c}
\hline \multicolumn{10}{c}{ Polygonal Pattern No. } \\
\hline \multicolumn{9}{c|}{$\mathrm{a}$} & \multicolumn{3}{c|}{$\mathrm{b}$} \\
\hline$r_{c}(\mathrm{~mm})$ & $N_{\text {side }}$ & $d_{s}(\mathrm{~mm})$ & $\psi(\mathrm{deg})$ & $r_{c}(\mathrm{~mm})$ & $N_{\text {side }}$ & $d_{s}(\mathrm{~mm})$ & $\psi(\mathrm{deg})$ & $r_{c}(\mathrm{~mm})$ & $N_{\text {side }}$ & $d_{s}(\mathrm{~mm})$ & $\psi(\mathrm{deg})$ \\
\hline 0.50 & 4 & 0.5 & 0 & 0.65 & 6 & 0.65 & 30 & 0.80 & 8 & 0.8 & 45 \\
\hline
\end{tabular}

circle radius $\left(r_{c}\right)$, number of sides $\left(N_{\text {side }}\right)$, and the helical angle $(\psi)$.
Fig. 12 shows the unit segments of the polygonal patterns mentioned in Table 8, the grinding wheels, and the related structured surfaces 


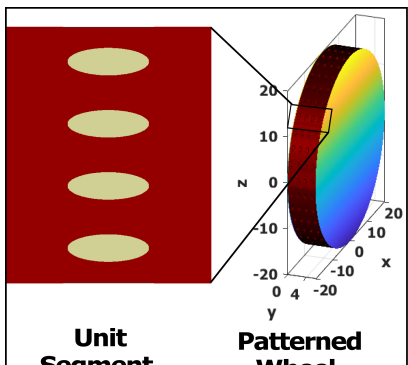
Wheel

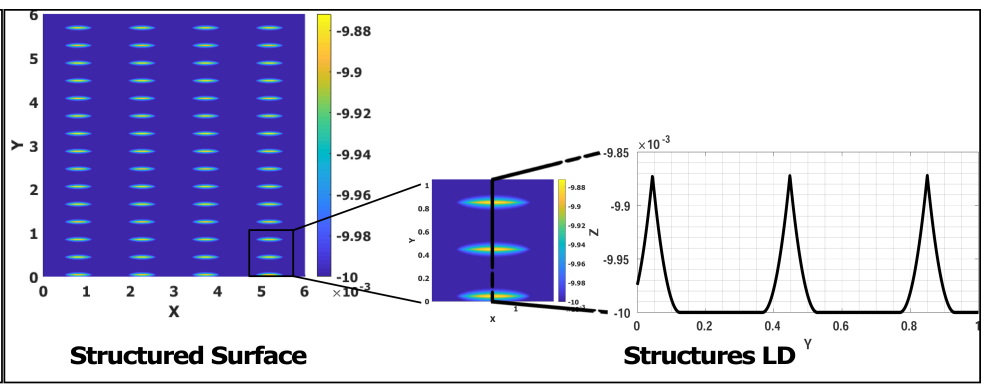

(a)

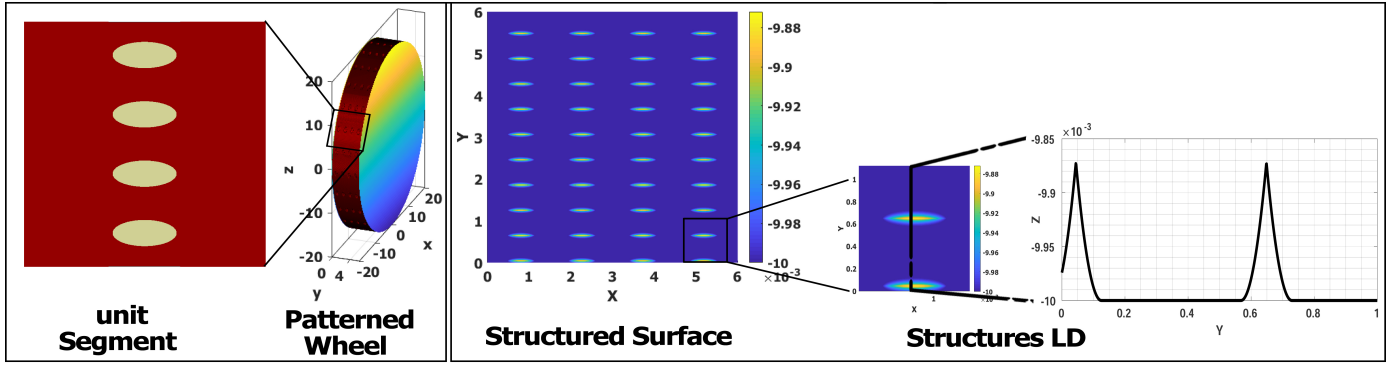

(b)
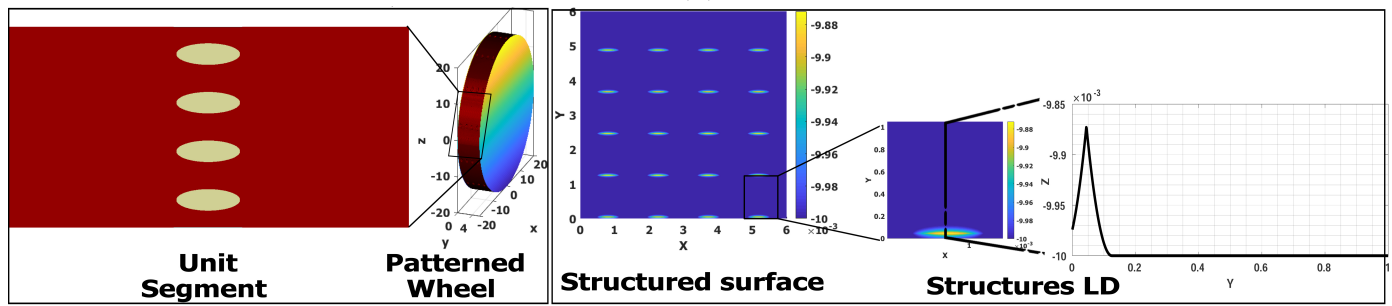

(c)

Fig. 11: The elliptical patterned wheels and the related structured surfaces at the increasing structuring ratio.

with $2 \mathrm{D}$ profiles across the structures $L D$. The differences in the size and the type between the designed patterned wheels are obvious in Fig. 12. With the increasing size of the polygonal pattern, the number of patterns and the structures $L D$ on the wheel surface decreases. Also, the circumferential distances and the circular pitches between the patterns on the wheel surface increase. As shown in Fig. 12, the geometry of the surface structure and the values of its geometrical parameters are affected by the pattern type and the pattern size of the grinding wheel. It can be noted on the surface structures and the enlarged views of them that the workpiece structures are reflections of the wheel patterns on the workpiece surface. The dimensions of the patterns on the wheel surface that are parallel to the grinding direction are scaled on the workpiece surface. But, the other dimensions that are normal to the grinding direction keep the same value with the patterns on the wheel surface. Table 9 includes the geometrical parameters of the structured surfaces shown in Fig. 12.

On the other side, the same procedures followed with the round patterns to illustrate the effect the changing structuring ratio $\left(\gamma_{s}\right)$ on the structured surface are tracked with the polygonal patterns. Fig. 13 displays the patterned wheels of hexagonal patterns designed 
Table 9: The geometrical parameters of the structured surfaces obtained by the polygonal patterned wheels

\begin{tabular}{|c|c|c|c|c|c|c|c|c|c|c|c|}
\hline \multicolumn{12}{|c|}{ The structured surface of polygonal pattern $\mathrm{No}$. } \\
\hline \multicolumn{4}{|c|}{$\mathrm{a}$} & \multicolumn{4}{|c|}{$\mathrm{b}$} & \multicolumn{4}{|c|}{$\mathrm{c}$} \\
\hline$S_{w}(m m)$ & $P(\mathrm{~mm})$ & $\vartheta($ deg $)$ & $S_{h}(\mu m)$ & $S_{w}(m m)$ & $P(\mathrm{~mm})$ & $\vartheta(\operatorname{deg})$ & $S_{h}(\mu m)$ & $S_{w}(m m)$ & $P(\mathrm{~mm})$ & $\vartheta($ deg $)$ & $S_{h}(\mu m)$ \\
\hline 0.167 & 0.199 & 0 & 0.850 & 0.217 & 0.349 & 5.50 & 0.294 & 0.248 & 0.491 & 9.46 & 0.745 \\
\hline
\end{tabular}

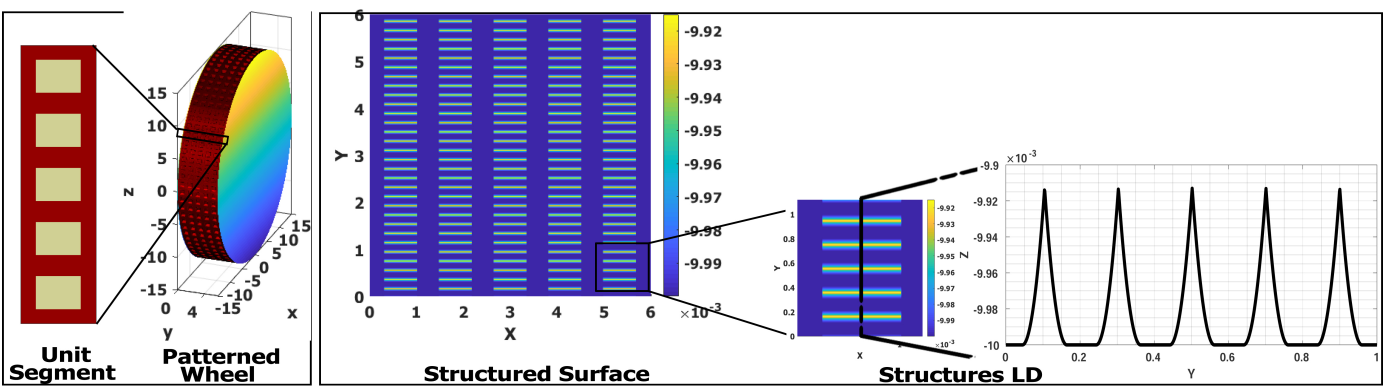

(a)

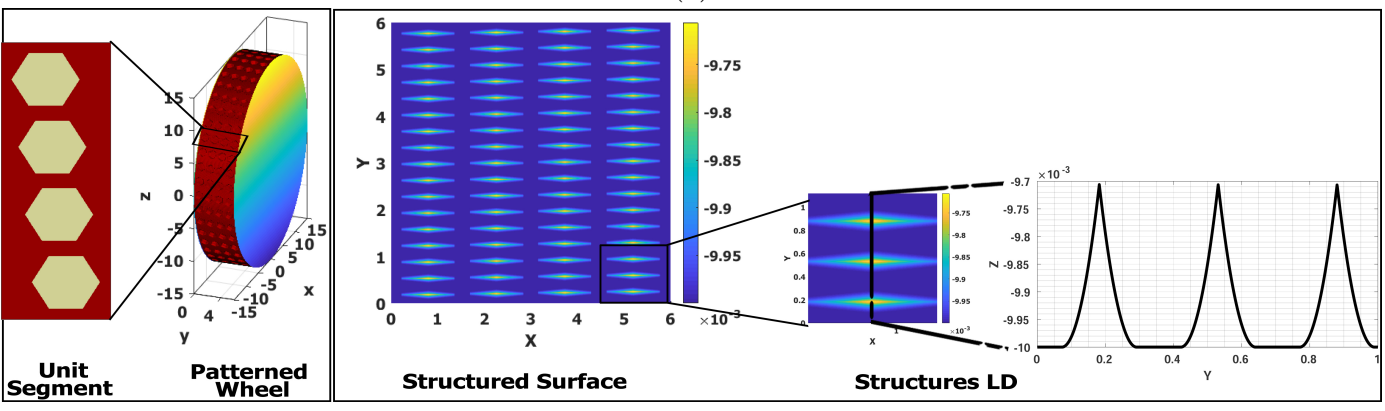

(b)
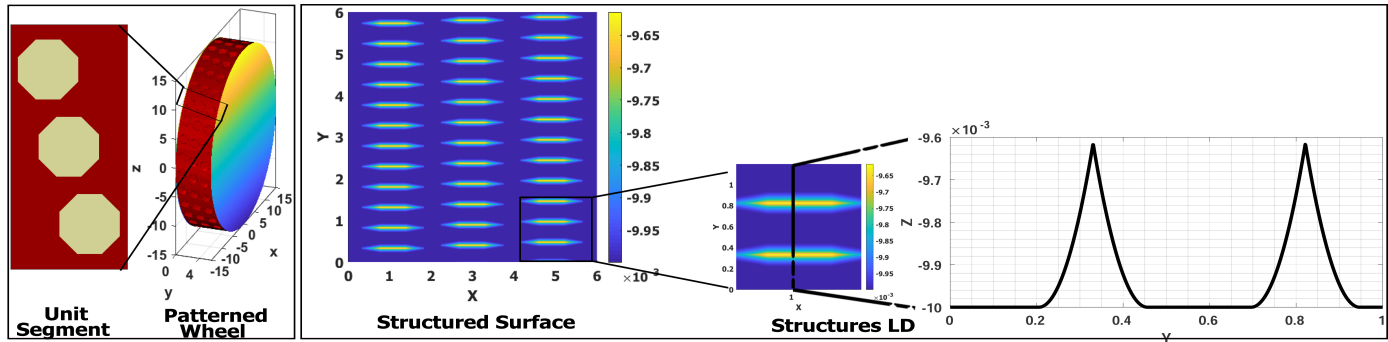

(c)

Fig. 12: The grinding wheels and the related structured surfaces with different polygonal patterns.

in Table 6 but with increasing value of the structuring ratio $\left(\gamma_{s}\right)$. The influence of the structuring ratio $\left(\gamma_{s}\right)$ of the wheel on the obtained structured surfaces is clear. The geometry of the structures on the workpiece surfaces shown in Fig. 13 is the same as that shown in Fig. 11b but at larger values of the longitudinal pitch $(P)$. This refers to the increasing circular pitch $\left(P_{c}\right)$ of the wheel patterns with the increasing structuring $\operatorname{ratio}\left(\gamma_{s}\right)$. See Eq. (11). The similarity in behavior of the structured surfaces at the analogous working conditions with the different types of wheel patterns proves the efficiency of the presented model for representing the structured surfaces with the specially designed patterned wheels. 


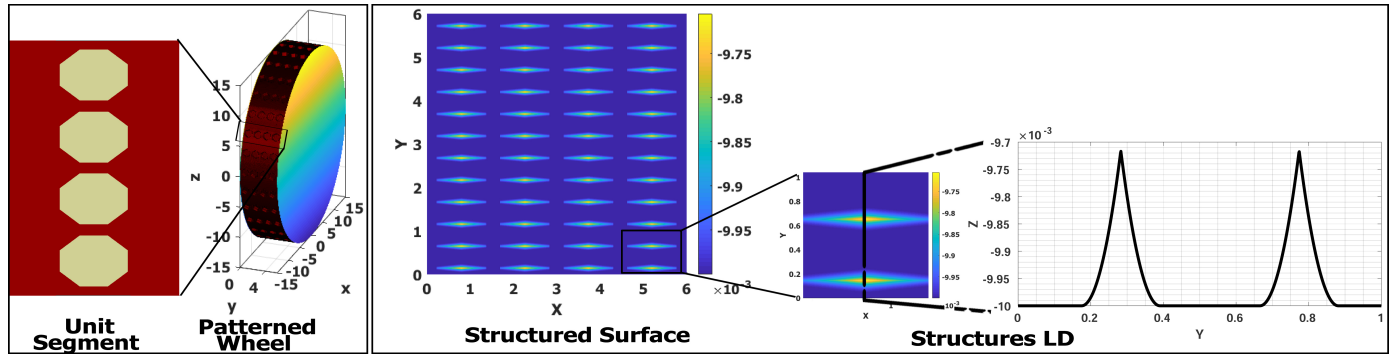

(a)
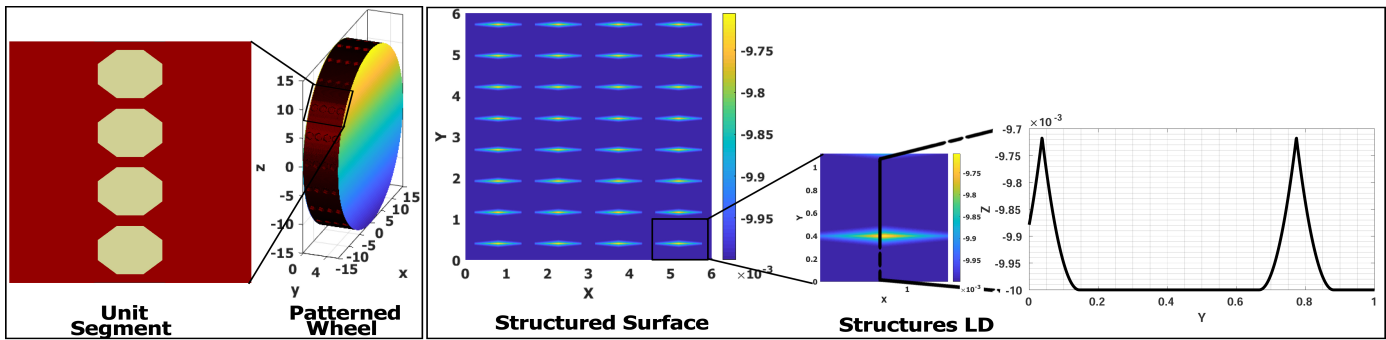

(b)
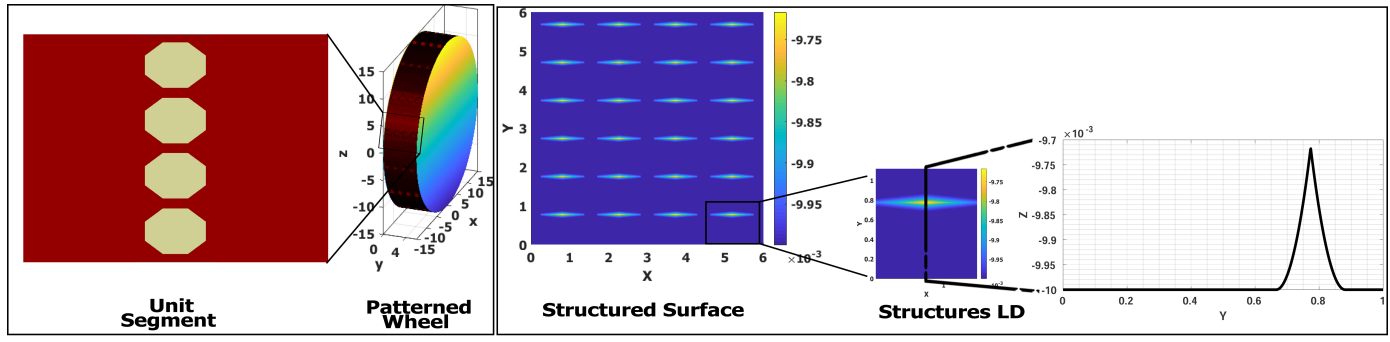

(c)

Fig. 13: The polygonal patterned wheels and the related structured surfaces at the increasing structuring ratio.

\subsection{Simulation of the structured surfaces} produced by structured wheels with irregular patterns

In addition to the regular geometries, grinding wheels with irregular patterned geometries and the corresponding surface structures can also be simulated. Unlike regular patterns, controlling the geometries of irregular patterns is to some extent more complex than regular ones. It can be done by changing and controlling different parameters of the driving formulas. See Eqs (8-9). In this research, a heart-like pattern is presented as an example for the irregularly patterned wheels with the corresponding structured surfaces in increasing size of the wheel pattern. Fig. 14 demonstrates the heartlike patterned wheels and the corresponding structured surfaces obtained at different pattern sizes at the same working conditions.

It can be shown that the behavior of the grinding wheel and the structured surface with the increasing size for the heart-like pattern is similar to those regular. As the pattern size increase, the number of patterns on the wheel surface decrease and the circular pitch $(P c)$ between them increase. Accordingly, the size of workpiece surface structures increases, and the structures $L D$ decreases with the increasing size of the wheel pattern. In addition, the pattern dimension parallel to the grinding di- 


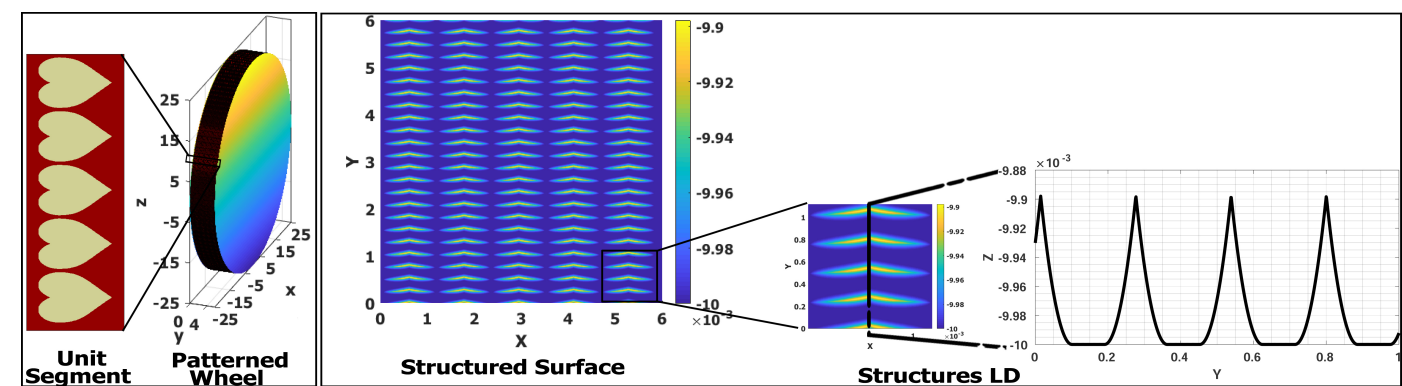

rection is scaled on the workpiece's structured
surface. While the other one normal to the

(a)
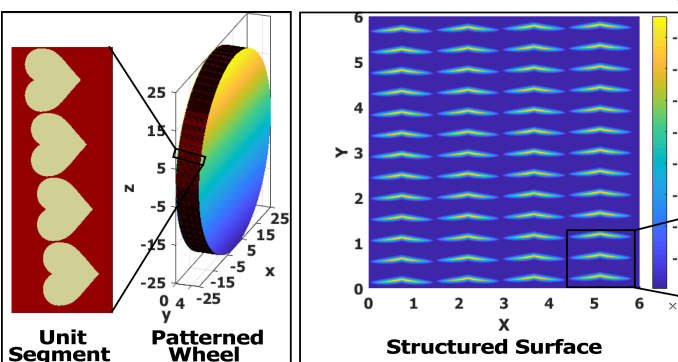

Structured Surface

Structures LD
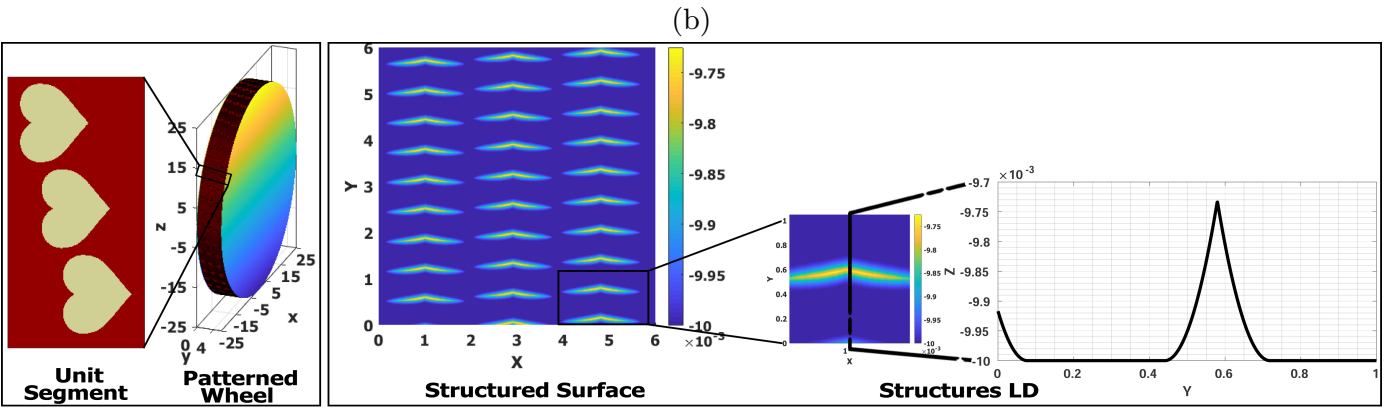

(c)

Fig. 14: The grinding wheels and the related structured surfaces at different heart-like pattern sizes. surfaces of the specially designed patterned wheels. grinding direction keeps the value of the pattern dimension (see Fig. 14). Fig. 15 shows the patterned wheels and the structured surface of the heart-like pattern with the increasing structuring ratio. Similarly, the surface structures have the same size at the increasing structuring ratio but with increasing longitudinal pitches and decreasing structures $L D$. The similarity of the results between regular and irregular patterned wheels can be considered evidence for the efficiency of the presented method for simulating and predicting the structured 

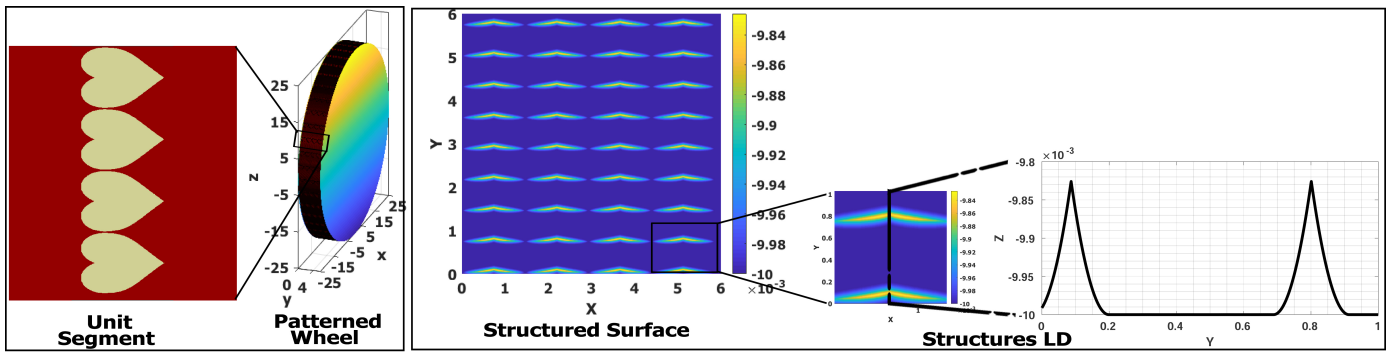

(a)
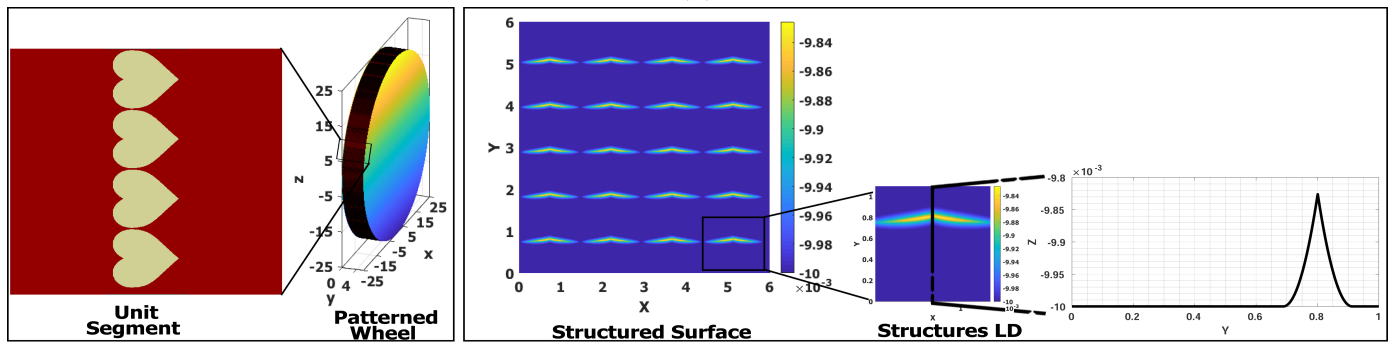

(b)
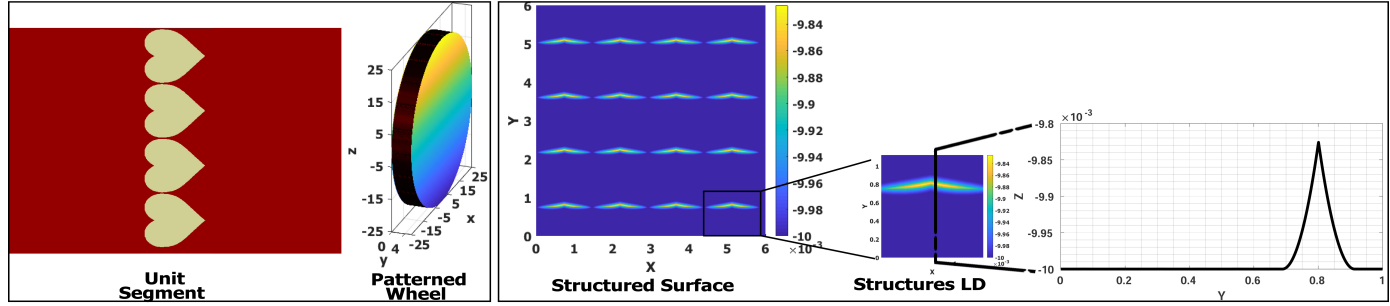

(c)

Fig. 15: The heart-like patterned wheels and the related structured surfaces at the increasing structuring ratio.

\section{Verification and Experimental Work}

In this section, both the ability and the accuracy of the presented model to predict and produce the machined structured surfaces are going to be measured and evaluated. As shown in Figure 16, MUGK7120X5 ultra-precision surface grinder has been constructed to be used as the experimental platform. A KAISER electric spindle was installed on the moving table of the surface grinder and it was adjusted to move along the $\mathrm{X}$-axis direction of the surface grinder. A laser displacement sensor was used for setting the axis eccentricity, the dynamic balance of the spindle was adjusted through the dynamic balance screws, and the spindle runs to $5000 R P M$ under the balanced condition. The balance deviation was set to be not more than $1 \mathrm{~nm}$ to ensure the stable operation of the spindle at high speed and to meet the requirements of precision grinding operations. After that, the structured grinding wheel was mounted on the electric spindle using a specially designed fixture, and the wheel runout and eccentricity have been adjusted. Then, tungsten carbide (WC) workpiece specimens have been mounted in position for performing the structuring processes on the machined surfaces. See Figure 17. To ensure the stability of the surface grinder machine tool in the precision grinding process, the actual feed rate used is not more than $1000 \mathrm{~mm} / \mathrm{min}$. 


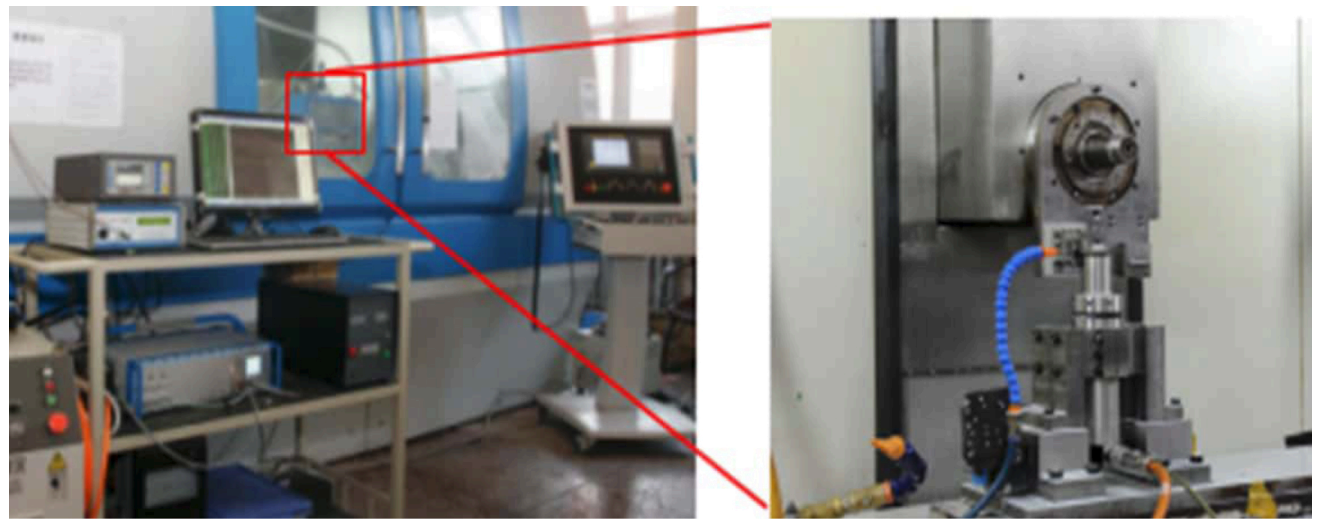

Fig. 16: The ultra-precision surface grinder.

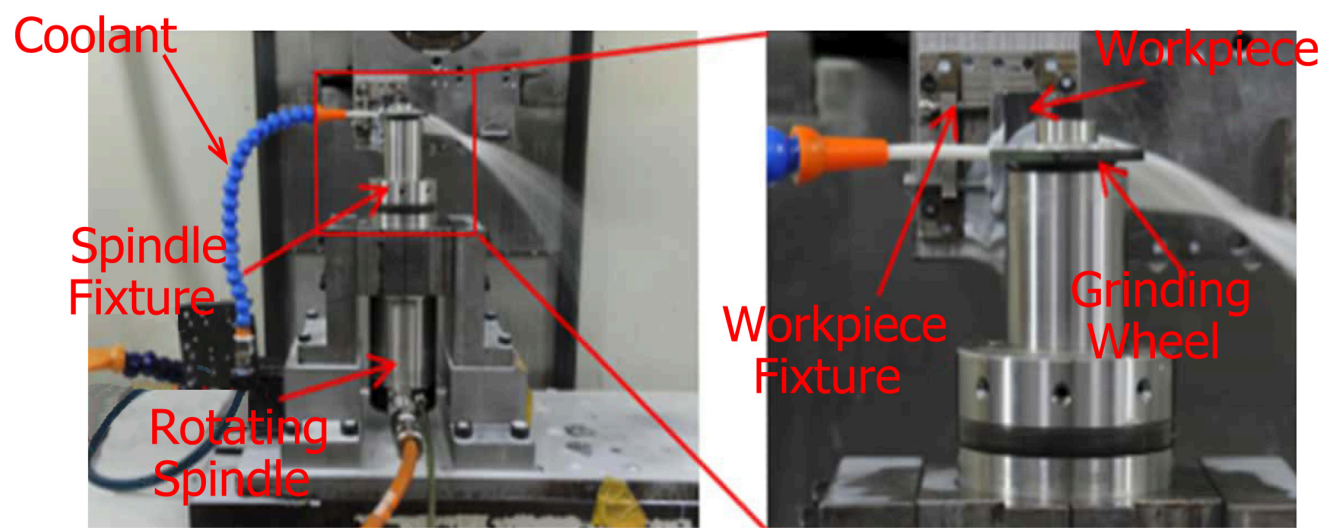

Fig. 17: (a) Components of the ultra-precision surface grinder, (b) Mounting of the grooved wheel and the workpiece specimens in position.

Figure 18 illustrates the geometry of the grinding wheel used in the current experimental work. A super abrasive diamond grinding wheel of $25 \mathrm{~mm}$ radius was prepared by pulsed
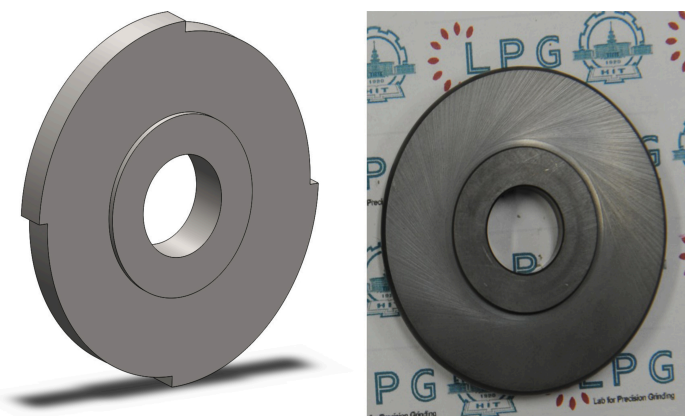

Fig. 18: The model and the real view of the grooved wheel used for carrying out experimental work $\left(N=2, \gamma_{s}=0.50, \psi=0 \mathrm{deg}\right)$. laser machining system and a motorized translation stage used for setting the grinding wheel in position to perform the laser structuring process. The grinding wheel is structured by

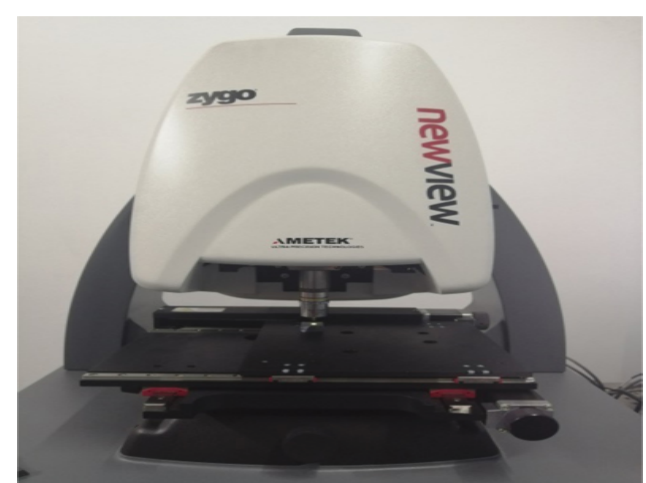

Fig. 19: The white light interferometer. 

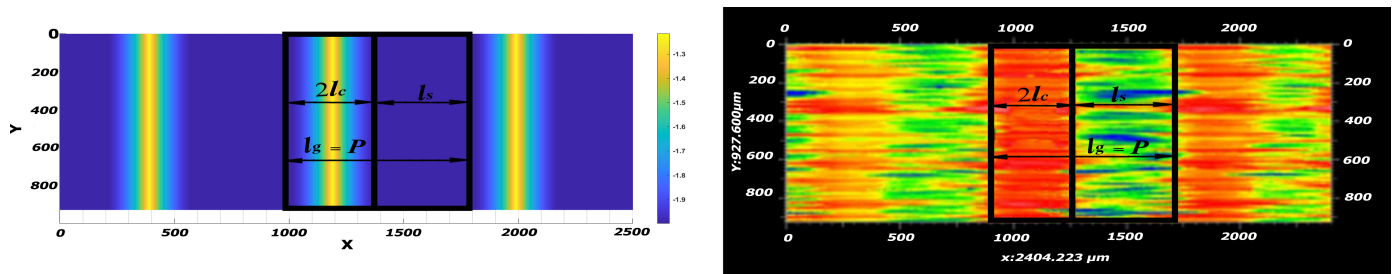

(a)
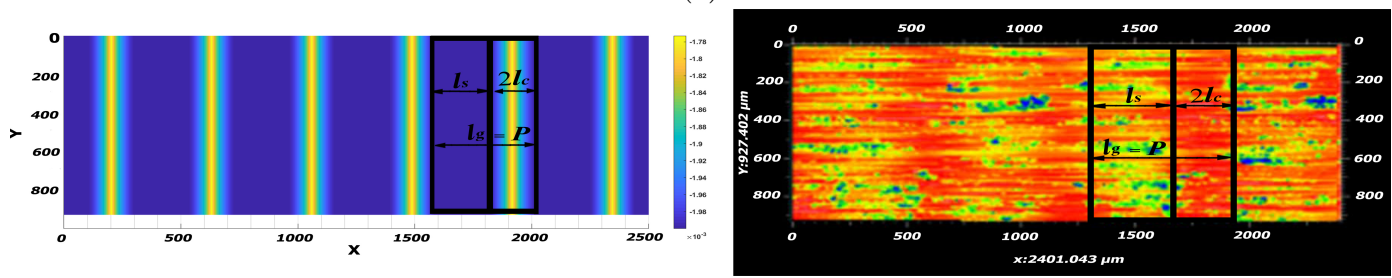

(b)
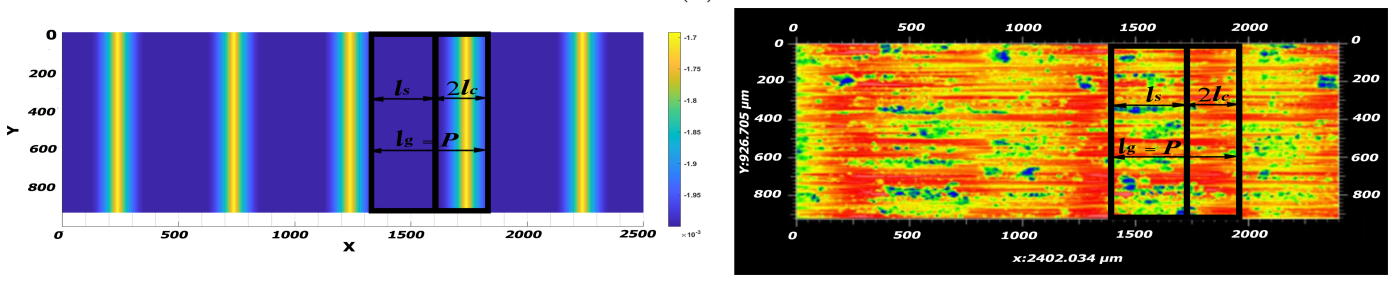

(c)
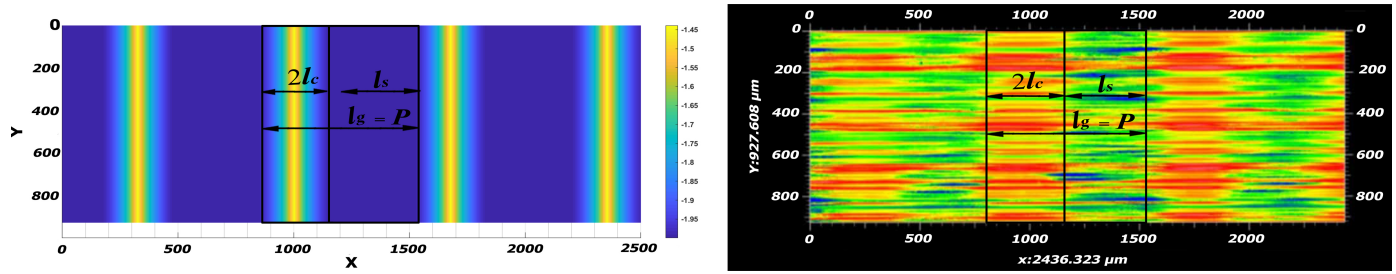

(d)

Fig. 20: The simulated and the experimental structured surfaces at the changing velocity ratio (dimensions in $\mu m$ ).

a ratio of $50 \%$ in two straight grooves to be used in performing the grinding processes.

In this work, two sets of grinding experiments have been designed. In the first set, the velocity ratio $\left(v^{*}\right)$ was varying by changing the values of working velocities while the grinding depth of cut $\left(d_{c}\right)$ was constant. For the other one, the depth of cut $\left(d_{c}\right)$ was changing and the velocity ratio $\left(v^{*}\right)$ was kept fixed. A white light interferometer (WLI) was used for inspection and analysis of the structured surfaces morphologies after executing the grinding operations. The device is illustrated in Figure 19.
Tables 10 and Table 11 include the values of the working parameters selected for both sets of experiments respectively. Figure 20 displays the simulated and the machined structured surfaces obtained at the working conditions listed in Table 10. The behavior of the longitudinal pitch with the changing velocity ratio is as expected. While the velocity ratio $\left(v^{*}\right)$ is decreasing (Figure 20 (a) to Figure 20 $(c))$, the pitch $(P)$ values decrease. When the velocity ratio $\left(v^{*}\right)$ increases again (Figure 20 $(\mathrm{d}))$, the pitch $(P)$ starts rising. Table 12 contains the values of the geometrical parameters 

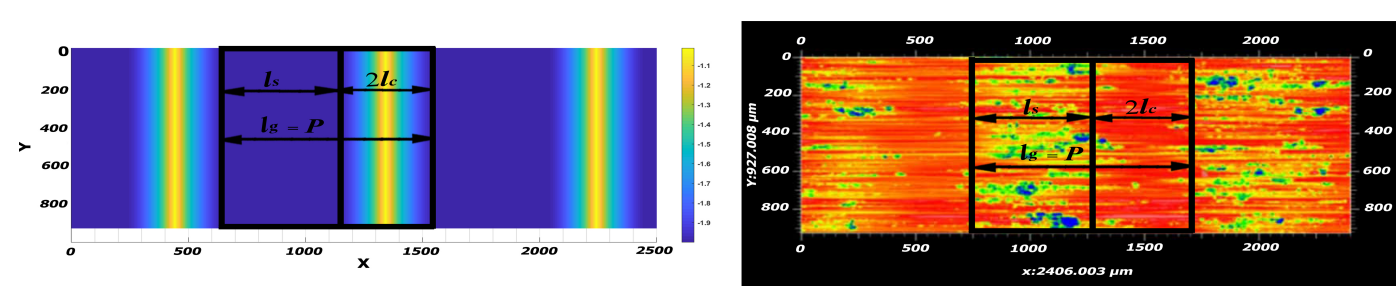

(a)
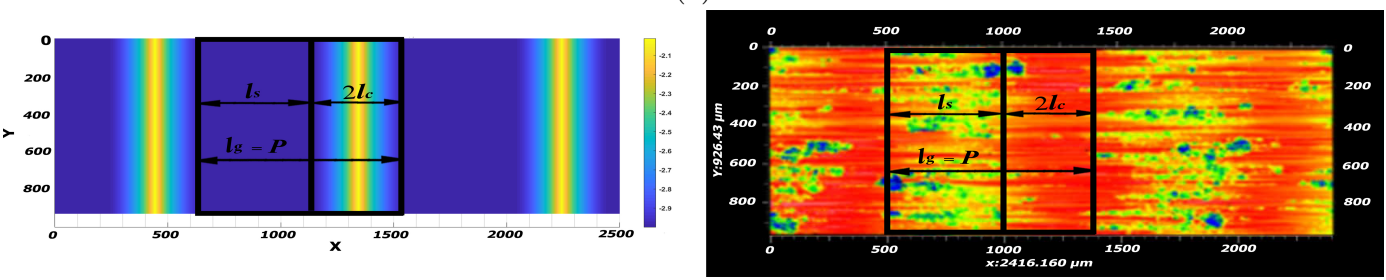

(b)
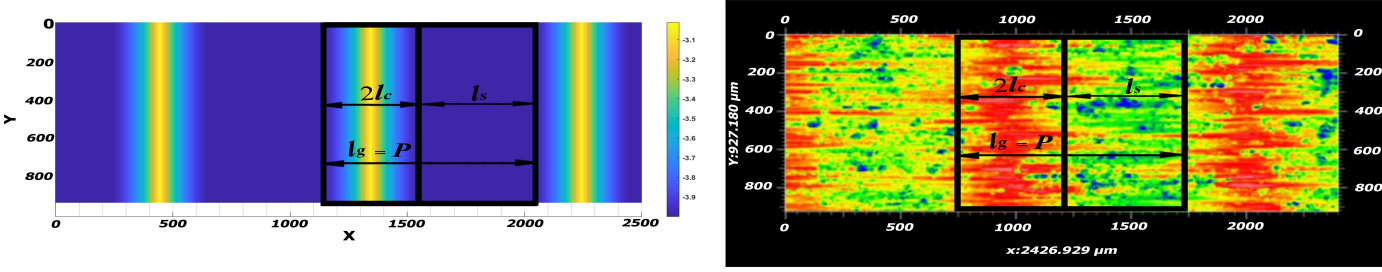

(c)
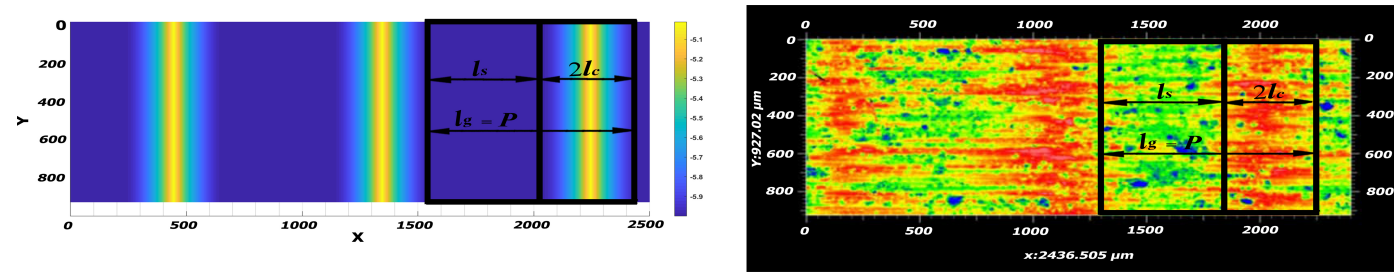

(d)

Fig. 21: The simulated and the machined surfaces at varying grinding depth (dimensions in $\mu m)$.

Table 10: Working conditions at the changing velocity ratio

\begin{tabular}{c|c|c|c|c}
\hline Experiment $N o$ & Wheel Velocity $R P M$ & Feed Rate $(\mathrm{mm} / \mathrm{min})$ & Velocity Ratio $v^{*}$ & Depth of cut $d_{c}(\mathrm{~mm})$ \\
\hline $\mathrm{a}$ & 500 & 800 & 0.0102 & 0.002 \\
\hline $\mathrm{b}$ & 600 & 700 & 0.0074 & 0.002 \\
\hline $\mathrm{c}$ & 700 & 700 & 0.0064 & 0.002 \\
\hline $\mathrm{d}$ & 500 & 676 & 0.0086 & 0.002 \\
\hline
\end{tabular}

Table 11: Working conditions at the changing depth of cut

\begin{tabular}{c|c|c|c|c}
\hline Experiment $N o$ & Wheel Velocity $R P M$ & Feed Rate $(\mathrm{mm} / \mathrm{min})$ & Velocity Ratio $v^{*}$ & Depth of cut $d_{c}(\mathrm{~mm})$ \\
\hline $\mathrm{a}$ & 500 & 900 & 0.0115 & 0.002 \\
\hline $\mathrm{b}$ & 500 & 900 & 0.0115 & 0.003 \\
\hline $\mathrm{c}$ & 500 & 900 & 0.0115 & 0.004 \\
\hline $\mathrm{d}$ & 500 & 900 & 0.0115 & 0.006 \\
\hline
\end{tabular}


Table 12: Values of the obtained simulated and machined geometrical parameters of the structured surfaces at different velocity ratios

\begin{tabular}{c|c|c|c|c|c|c}
\hline \multirow{2}{*}{$N o$} & \multicolumn{2}{|c|}{ Straight Width $l_{s}(\mathrm{~mm})$} & \multicolumn{2}{c|}{ Curved Width $l_{c}$} & \multicolumn{2}{c}{ Pitch $P$} \\
\cline { 2 - 7 } & Simulation & Machining & Simulation & Machining & Simulation & Machining \\
\hline $\mathrm{a}$ & 0.485 & 0.460 & 0.1575 & 0.185 & 0.800 & 0.830 \\
\hline $\mathrm{b}$ & 0.390 & 0.430 & 0.0975 & 0.100 & 0.585 & 0.630 \\
\hline $\mathrm{c}$ & 0.295 & 0.330 & 0.1025 & 0.110 & 0.500 & 0.550 \\
\hline $\mathrm{d}$ & 0.338 & 0.355 & 0.169 & 0.1775 & 0.676 & 0.710 \\
\hline
\end{tabular}

Table 13: The simulated and the machined surfaces at varying grinding depth (dimensions in $\mu \mathrm{m})$.

\begin{tabular}{c|c|c|c|c|c|c}
\hline \multirow{2}{*}{$N o$} & \multicolumn{2}{|c|}{ Straight Width $l_{s}(\mathrm{~mm})$} & \multicolumn{2}{c|}{ Curved Width $l_{c}$} & \multicolumn{2}{c}{ Pitch $P$} \\
\cline { 2 - 7 } & Simulation & Machining & Simulation & Machining & Simulation & Machining \\
\hline $\mathrm{a}$ & 0.500 & 0.530 & 0.200 & 0.210 & 0.900 & 0.930 \\
\hline $\mathrm{b}$ & 0.520 & 0.500 & 0.190 & 0.190 & 0.900 & 0.880 \\
\hline $\mathrm{c}$ & 0.525 & 0.530 & 0.1625 & 0.225 & 0.900 & 0.980 \\
\hline $\mathrm{d}$ & 0.540 & 0.550 & 0.180 & 0.200 & 0.900 & 0.950 \\
\hline
\end{tabular}

for the simulated and the machined surfaces shown in Figure 20.

Figure 21 visualizes the obtained structured surfaces using the working conditions listed in Table 11 at the changing grinding depth $\left(d_{c}\right)$. Table 13 contains the values of the obtained geometries at the second set of the grinding conditions. As the speed ratio $\left(v^{*}\right)$ was constant, the pitch values for the simulated pitches $P$ are constant. A satisfied agreement can be noted between the simulated and the machined values for the geometrical parameters of the structured surfaces for both experiments sets.

\section{Conclusion}

In this paper, the ability for machining the structured surfaces using specially designed patterned wheels has been studied. Several regular and irregular patterns have been applied. The influences of the wheel patterns on the produced structured surfaces has been demonstrated. The conclusions are as follows:

1. Machining of the structured surfaces by the grinding method using different geometries for the wheel patterns rather than the straight/helical patterns is applicable. Different regular and irregular patterns such as circular, elliptical, polygonal, and heart shapes at different working conditions are formulated and simulated.

2. With the increasing wheel pattern size, the number of patterns on the wheel surface decreases but the circumferential distances separating the patterns on the wheel surface increase.

3. As the size of the wheel pattern increases, the geometrical parameters of the workpiece structure increase and number of structures for the unit length decreases. Both the structure width and the structure height increase and the longitudinal distances between structures increases with the increasing pattern size.

4. At constant wheel pattern size, the structuring ratio of the grinding wheels control the structures linear density through adjusting the longitudinal pitch between structures without affecting the size of the surface structure itself.

5. Dimensions of the wheel patterns parallel to the grinding direction is scaled on the workpiece surface unlike that is normal to the grinding direction which dont differ from those on the wheel.

- Ethical Approval All procedures performed in studies involving human participants were in accordance with the ethical standards of the institutional and/or national research 
committee and with the 1964 Helsinki declaration and its later amendments or comparable ethical standards. This article does not contain any studies with human participants or animals performed by any of the authors.

- Consent to Participate Informed consent was obtained from all individual participants included in the study.

- Consent to Publish Informed consent was obtained from all individual participants included in the study.

- Authors Contributions 1. Amr Monier: Conceptualization, Methodology, Investigation, Software, Validation, Data curation, Supervision, Formal analysis, Writing4. original draft, Writing-reviewing \& editing.

2. Bing Guo: Methodology, Software, Resources, Supervision, Funding acquisition, Project administration, Validation, Resources, Reviewing-original draft.

3. Qingliang Zhao: Resources, Supervision, Funding acquisition, Project administration, Validation Visualization, Measurements, Formal analysis, Reviewingoriginal draft.

4. T.S.Mahmoud: Formal analysis, Investigation, Data curation ,Validation, Reviewing-original draft.

- Funding This work was supported by National Natural Scientific Foundation of China [Grant No.: 51405108], Enterprise Innovation and Development Joint Program of the National Natural Science Foundation of China [Grant No.: U20B2032].

- Competing Interests The authors declare that they have no known competing financial interests or personal relationships that could have appeared to influence the work reported in this paper. Availability of data and materials The manuscrajpeng, Se-Woong Baek, Ji-Won Seo, has not been published previously (partly or in full) elsewhere.

\section{References}

1. AAG Bruzzone, HL Costa, PM Lonardo, and DA Lucca. Advances in engineered surfaces for functional performance. CIRP annals, 57(2):750-769, 2008.

2. Leonardo De Chiffre, H Kunzmann, GN Peggs, and DA Lucca. Surfaces in precision engineering, microengineering and nanotechnology. CIRP Annals, 52(2):561577, 2003.

3. Lidia Galda, Pawel Pawlus, and Jaroslaw Sep. Dimples shape and distribution effect on characteristics of stribeck curve. Tribology International, 42(10):1505-1512, 2009. T Ibatan, MS Uddin, and MAK Chowdhury. Recent development on surface texturing in enhancing tribological performance of bearing sliders. Surface and Coatings Technology, 272:102-120, 2015.

5. HL Costa and IM Hutchings. Hydrodynamic lubrication of textured steel surfaces under reciprocating sliding conditions. Tribology International, 40(8):12271238, 2007.

6. HL Costa and IM Hutchings. Effects of die surface patterning on lubrication in strip drawing. Journal of Materials Processing Technology, 209(3):1175-1180, 2009.

7. B Denkena, D Boehnke, C Spille, and $\mathrm{R}$ Dragon. In-process information storage on surfaces by turning operations. CIRP annals, 57(1):85-88, 2008.

8. Fengyou Wang, Xiaodan Zhang, Liguo Wang, Yuanjian Jiang, Changchun Wei, and Ying Zhao. Pyramidal texturing of silicon surface via inorganic--organic hybrid alkaline liquor for heterojunction solar cells. Journal of Power Sources, 293:698705, 2015.

9. Changsoon Cho, Hoyeon Kim, Seonju Donggeon Han, Kyoohyun Kim, YongKeun Park, Seunghyup Yoo, and Jung-Yong Lee. Random and v-groove texturing for efficient light trapping in organic photovoltaic cells. Solar energy 
materials and solar cells, 115:36-41, 2013.

10. Jinyu Zhang and Yonggang Meng. A study of surface texturing of carbon steel by photochemical machining. Journal of Materials Processing Technology, 212(10):21332140, 2012.

11. Hao Nan Li, Yue Yang, Yong Jie Zhao, Zhanlin Zhang, Weiqiang Zhu, Weiling Wang, and Huan Qi. On the periodicity of fixed-abrasive planetary lapping based on a generic model. Journal of Manufacturing Processes, 44:271-287, 2019.

12. Feihu Zhang, Binbin Meng, Yanquan Geng, and Yong Zhang. Study on the machined depth when nanoscratching on 6h-sic using berkovich indenter: Modelling and experimental study. Applied Surface Science, 368:449-455, 2016.

13. Bing Guo, Qingliang Zhao, and Xin Yu. Surface micro-structuring of coarsegrained diamond wheels by nanosecond pulsed laser for improving grinding performance. International journal of precision engineering and manufacturing, 15(10):2025-2030, 2014.

14. Hao Nan Li and Dragos Axinte. Textured grinding wheels: a review. International Journal of Machine Tools and Manufacture, 109:8-35, 2016.

15. Cameron Forbrigger, Robert Bauer, and Andrew Warkentin. A review of stateof-the-art vitrified bond grinding wheel grooving processes. The International Journal of Advanced Manufacturing Technology, 90(5):2207-2216, 2017.

16. XH Zhang, ZX Kang, S Li, QP Wu, and ZC Zhang. Experimental investigations on the impact of different laser macrostructured diamond grinding wheels on alumina ceramic. The International Journal of Advanced Manufacturing Technology, 96(5):1959-1969, 2018.

17. Hui Deng and Jie He. A study of the grinding performance of laser microstructured coarse-grained diamond grinding wheels. The International Journal of Advanced Manufacturing Technology,
93(5):1989-1997, 2017.

18. Piotr Stępień and Maciej Szafarczyk. Generation of regular patterns on ground surfaces. CIRP annals, 38(1):561-566, 1989.

19. Piotr Stępień. Regular surface texture generated by special grinding process. Journal of manufacturing science and engineering, 131(1), 2009.

20. Berend Denkena, Jens Köhler, and Bo Wang. Manufacturing of functional riblet structures by profile grinding. CIRP Journal of Manufacturing Science and Technology, 3(1):14-26, 2010.

21. JFG Oliveira, AC Bottene, and TV Franca. A novel dressing technique for texturing of ground surfaces. CIRP annals, 59(1):361-364, 2010.

22. Eraldo Jannone da Silva, João Fernando Gomes de Oliveira, Bruno Barbosa Salles, Ricardo Santoro Cardoso, and Vinicius Ricco Alves Reis. Strategies for production of parts textured by grinding using patterned wheels. CIRP Annals, 62(1):355-358, 2013.

23. AL-Mokhtar O Mohamed, Andrew Warkentin, and Robert Bauer. Prediction of workpiece surface texture using circumferentially grooved grinding wheels. The International Journal of Advanced Manufacturing Technology, 89(1-4):1149-1160, 2017.

24. Yueming Liu, Andrew Warkentin, Robert Bauer, and Yadong Gong. Investigation of different grain shapes and dressing to predict surface roughness in grinding using kinematic simulations. Precision Engineering, 37(3):758-764, 2013.

25. Bruce E Meserve. Fundamental concepts of geometry. Courier Corporation, 2014. 